Geometry $\&$ Topology

Volume 9 (2005) 1443-1499

Published: 8 August 2005

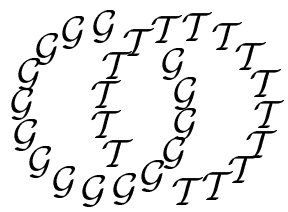

\title{
Khovanov's homology for tangles and cobordisms
}

\author{
Dror BAR-NATAN \\ Department of Mathematics, University of Toronto \\ Toronto, Ontario M5S 3G3, Canada \\ Email: drorbn@math.toronto.edu \\ URL: http://www.math.toronto.edu/ drorbn
}

\begin{abstract}
We give a fresh introduction to the Khovanov Homology theory for knots and links, with special emphasis on its extension to tangles, cobordisms and 2-knots. By staying within a world of topological pictures a little longer than in other articles on the subject, the required extension becomes essentially tautological. And then a simple application of an appropriate functor (a "TQFT") to our pictures takes them to the familiar realm of complexes of (graded) vector spaces and ordinary homological invariants.
\end{abstract}

\section{AMS Classification numbers Primary: 57M25}

Secondary: 57M27

Keywords: 2-knots, canopoly, categorification, cobordism, Euler characteristic, Jones polynomial, Kauffman bracket, Khovanov, knot invariants, movie moves, planar algebra, skein modules, tangles, trace groups

Proposed: Vaughan Jones

Seconded: Robion Kirby, Cameron Gordon
Received: 3 November 2004

Accepted: 04 July 2005 


\section{Introduction}

The Euler characteristic of a space is a fine invariant; it is a key ingredient in any discussion of the topology of surfaces (indeed, it separates closed orientable surfaces), and it has further uses in higher dimensions as well. But homology is way better. The alternating sum of the ranks of the homology groups of a space is its Euler characteristic, so homology is at least as strong an invariant, and it is easy to find examples showing that homology is a strictly stronger invariant than the Euler characteristic.

And then there's more. Unlike the Euler characteristic, homology is a functor - continuous maps between spaces induce maps between their homologies, and it is this property of homology that makes it one of the cornerstones of algebraic topology; not merely the fact that it is a little better then the Euler characteristic at telling spaces apart.

In his seminal paper [10] Khovanov explained that the Jones polynomial of a link $L$ is the (graded) Euler characteristic of a certain "link homology" theory. In my follow up paper [3] I have computed the Khovanov homology of many links and found that indeed it is a stronger invariant than the Jones polynomial, as perhaps could be expected in the light of the classical example of the Euler characteristic and the homology of spaces.

In further analogy with the classical picture, Jacobsson [6] and Khovanov [13] found what seems to be the appropriate functoriality property of the Khovanov homology. What replaces continuous maps between spaces is $4 \mathrm{D}$ cobordisms between links: Given such a cobordism $C$ between links $L_{1}$ and $L_{2}$ Jacobsson and Khovanov show how to construct a map $K h(C): K h\left(L_{1}\right) \rightarrow K h\left(L_{2}\right)$ (defined up to a \pm sign) between the corresponding Khovanov homology groups $K h\left(L_{1}\right)$ and $K h\left(L_{2}\right) 1$ Note that if $L_{1}$ and $L_{2}$ are both the empty link, then a cobordism between $L_{1}$ and $L_{2}$ is a $2-$ knot in $\mathbb{R}^{4}$ (see eg, [4) and $K h(C): K h\left(L_{1}\right) \rightarrow K h\left(L_{2}\right)$ becomes a group homomorphism $\mathbb{Z} \rightarrow \mathbb{Z}$, hence a single scalar (defined up to a sign). That is, this special case of $K h(C)$ yields a numerical invariant of $2-\operatorname{knots}{ }^{2}$

Given a "movie presentation" of a cobordism $C$ (as in [4), the construction of $K h(C)$ is quite simple to describe. But the proofs that $K h(C)$ is independent of the specific movie presentation chosen for $C$ are quite involved. Jacobsson's proof involves a large number of complicated case by case computations.

\footnotetext{
${ }^{1}$ Check [16] for a topological application and [5] for some computations.

${ }^{2}$ Added July 2005: The latter is trivial; see [17].
} 
Khovanov's proof is more conceptual, but it relies on his rather complicated "functor-valued invariant of tangles" [11] and even then there remains some case-checking to do.

A major purpose of this article is to rewrite Khovanov's proof in a simpler language. Thus a major part of our work is to simplify (and at the same time extend!) Khovanov's treatment of tangles. As side benefits we find a new homology theory for knots/links (Section 9.3) and what we believe is the "right" way to see that the Euler characteristic of Khovanov homology is the Jones polynomial (Section 10).

\section{$1.1 \quad$ Executive summary}

This quick section is for the experts. If you aren't one, skip straight to Section 1.2

\subsubsection{Why are tangles relevant to cobordisms?}

Tangles are knot pieces, cobordisms are movies starring knots and links [4]. Why is the former relevant to the study of the latter (in the context of Khovanov homology)?

The main difficulty in showing that cobordisms induce maps of homology groups is to show that trivial movies induce trivial maps on homology. A typical example of such a trivial movie is the circular clip

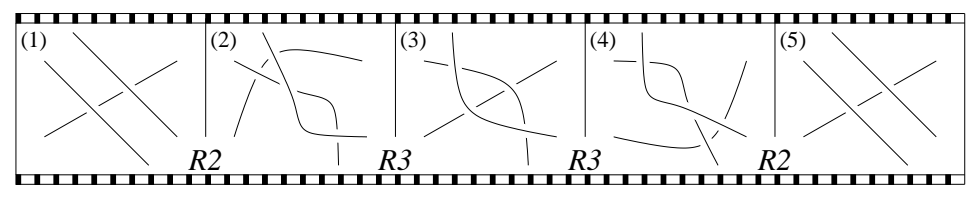

(this is $\mathrm{MM}_{6}$ of Figure 12). Using a nice theory for tangles which we will develop later, we will be able to replace the composition of morphisms corresponding to the above clip by the following composition, whose "core" (circled below) 
remains the same as in clip (1):

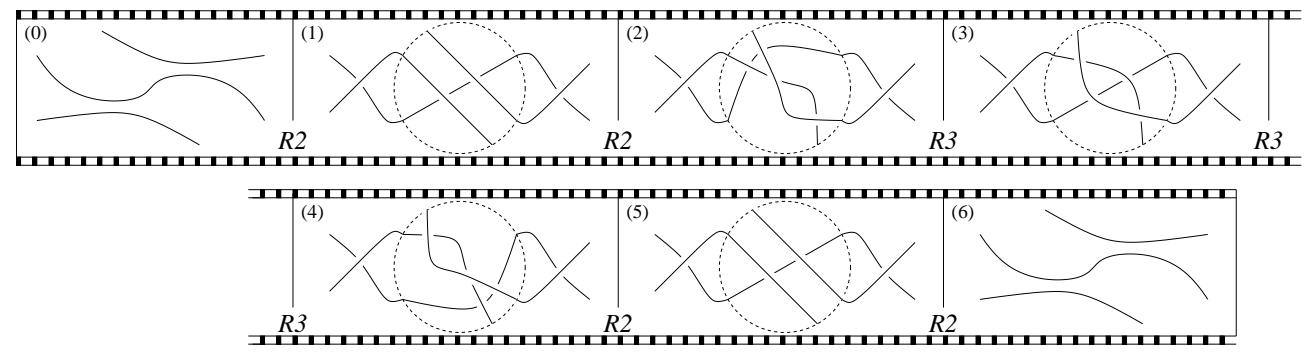

But this composition is an automorphism of the complex $K$ of the crossingless tangle $T=\smile$ and that complex is very simple - as $T$ has no crossings, $K$ ought td 3 consist only of one chain group and no differentials. With little luck, this would mean that $K$ is also simple in a technical sense - that it has no automorphisms other than multiples of the identity. Thus indeed the circular clip of Equation (10) induces a trivial (at least up to a scalar) map on homology.

In the discussion of the previous paragraph it was crucial that the complex for the tangle $T=\smile$ be simple, and that it would be possible to manipulate tangles as in the transition from (11) to (2). Thus a "good" theory of tangles is useful for the study of cobordisms.

\subsubsection{How we deal with tangles}

As defined in [10] (or in [3] or in [21]), the Khovanov homology theory does not lend itself naturally to an extension to tangles. In order to define the chain spaces one needs to count the cycles in each smoothing, and this number is not known unless all 'ends' are closed, ie, unless the tangle is really a link. In 11 Khovanov solves the problem by taking the chain space of a tangle to be the direct sum of all chain spaces of all possible closures of that tangle. Apart from being quite cumbersome (when all the details are in place; see [11]), as written, Khovanov's solution only allows for 'vertical' compositions of tangles, whereas one would wish to compose tangles in arbitrary planar ways, in the spirit of V Jones' planar algebras [8].

We deal with the extension to tangles in a different manner. Recall that the Khovanov picture of [10] can be drawn in two steps. First one draws a 'topological picture' Top made of smoothings of a link diagram and of cobordisms

\footnotetext{
${ }^{3}$ We say "ought to" only because at this point our theory of tangles is not yet defined
} 
between these smoothings. Then one applies a certain functor $\mathcal{F}(\mathrm{a}(1+1)-$ dimensional TQFT) to this topological picture, resulting in an algebraic picture $\mathcal{A l g}$, a complex involving modules and module morphisms whose homology is shown to be a link invariant. Our trick is to postpone the application of $\mathcal{F}$ to a later stage, and prove the invariance already at the level of the topological picture. To allow for that, we first need to 'mod out' the topological picture by the 'kernel' of the 'topology to algebra' functor $\mathcal{F}$. Fortunately it turns out that that 'kernel' can be described in completely local terms and hence our construction is completely local and allows for arbitrary compositions. For the details, read on.

\subsection{The plan}

A traditional math paper sets out many formal definitions, states theorems and moves on to proving them, hoping that a "picture" will emerge in the reader's mind as (s)he struggles to interpret the formal definitions. In our case the "picture" can be summarized by a rather fine picture that can be uploaded to one's mind even without the formalities, and, in fact, the formalities won't necessarily make the upload any smoother. Hence we start our article with the picture, Figure 1, and follow it in Section 2 by a narrative description thereof, without yet assigning any meaning to it and without describing the "frame" in which it lives - the category in which it is an object. We fix that in Sections 3 and 4 in the former we describe a certain category of complexes where our picture resides, and in the latter we show that within that category our picture is a homotopy invariant. The nearly tautological Section 5 discusses the good behaviour of our invariant under arbitrary tangle compositions. In Section 6 we refine the picture a bit by introducing gradings, and in Section 7 we explain that by applying an appropriate functor $\mathcal{F}($ a $1+1$-dimensional TQFT) we can get a computable homology theory which yields honest knot/link invariants.

While not the technical heart of this paper, Sections 810 are its raison d'être. In Section 8 we explain how our machinery allows for a simple and conceptual explanation of the functoriality of the Khovanov homology under tangle cobordisms. In Section 9 we further discuss the "frame" of Section 3 finding that in the case of closed tangles (ie, knots and links) and over rings that contain $\frac{1}{2}$ it frames very little beyond the original Khovanov homology while if 2 is not invertible our frame appears richer than the original. In Section 10] we introduce a generalized notion of Euler characteristic which allows us to "localize" the assertion "The Euler characteristic of Khovanov Homology is the Jones polynomial". 
The final Section 11 contains some further "odds and ends".

\subsection{Acknowledgement}

I wish to thank B Chorny, L Kauffman, M Khovanov, A Kricker, G Naot, J Przytycki, A Referee, J Roberts, SD Schack, K Shan, A Sikora, A Shumakovich, J Stasheff, D Thurston and Y Yokota for their comments and suggestions and S Carter and M Saito for allowing me to use some figures from 4 . This work was partially supported by NSERC grant RGPIN 262178.

\section{A picture's worth a thousand words}

As promised in the introduction — we'd like to start with Figure 1 at a completely descriptive level. All interpretations will be postponed to later sections.

2.1 Knot On the upper left of the figure we see the lefthanded trefoil knot $K$ with its $n=3$ crossings labeled 1, 2 and 3 . It is enclosed in double brackets $([\cdot])$ to indicate that the rest of the figure shows the formal Khovanov Bracket of

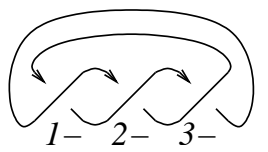
the left-handed trefoil. As we describe the rest of the figure we will also indicate how it changes if the left-handed trefoil is replaced by an arbitrary other knot or link.

2.2 Crossings On the figure of $K$ we have also marked the signs of its crossings - $(+)$ for overcrossings ( $\times)$ and $(-)$ for

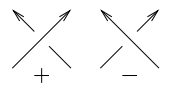
undercrossings $\left(\lambda^{\top}\right)$. Let $n_{+}$and $n_{-}$be the numbers of $(+)$crossings and $(-)$ crossings in $K$, respectively. Thus for the left-handed trefoil knot, $\left(n_{+}, n_{-}\right)=$ $(0,3)$.

2.3 Cube The main part of the figure is the 3dimensional cube whose vertices are all the 3 -letter strings of 0 's and 1's. The edges of the cube are marked in the natural manner by 3 -letter strings of 0 's, 1 's and precisely one $\star$ (the $\star$ denotes the

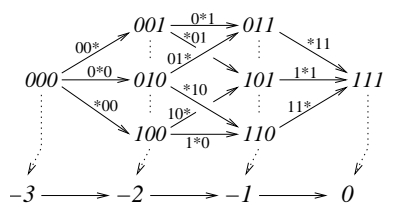
coordinate which changes from 0 to 1 along a given edge). The cube is skewered along its main diagonal, from 000 to 111. More precisely, each vertex of the cube has a "height", the sum of its coordinates, a number between 0 and 3 . The cube is displayed in such a way so that vertices of height $k$ project down to the point $k-n_{-}$on a line marked below the cube. We've indicated these projections with dashed arrows and tilted them a bit to remind us of the $-n_{-}$ shift. 


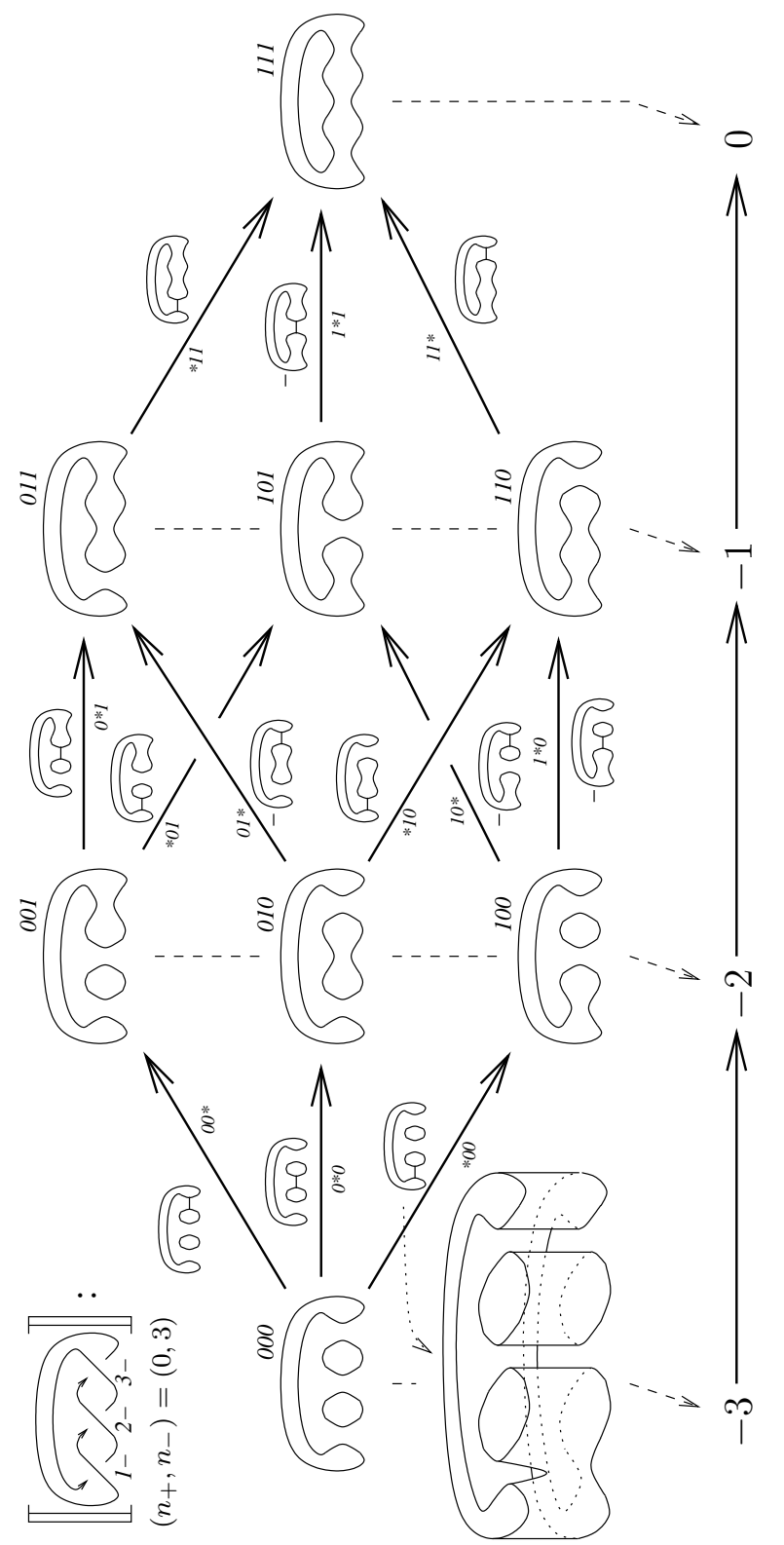

Figure 1: The main picture. See the narrative in Section 2 

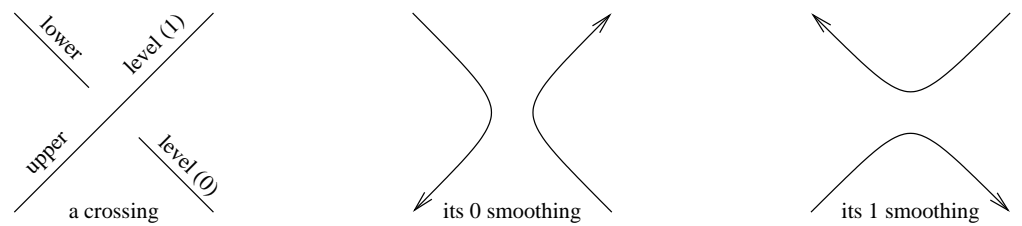

Figure 2: A crossing is an interchange involving two highways. The 0 -smoothing is when you enter on the lower level (level 0 ) and turn right at the crossing. The 1 -smoothing is when you enter on the upper level (level 1) and turn right at the crossing.

2.4 Aside: More crossings Had we been talking about some $n$ crossing knot rather than the 3-crossing left-handed trefoil, the core of our picture would have been the $n$ dimensional cube with vertices $\{0,1\}^{n}$, projected by the "shifted height" to the integer points on the interval $\left[-n_{-}, n_{+}\right]$.

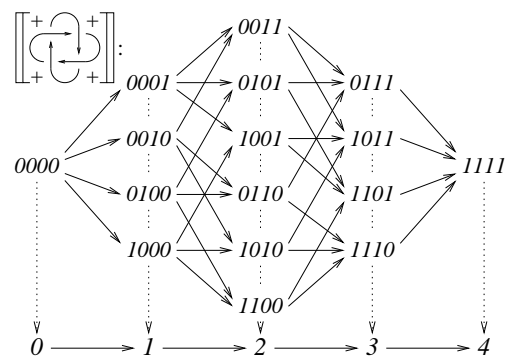

2.5 Vertices Each vertex of the cube carries a smoothing of $K$ - a planar diagram obtained by replacing every crossing $\times$ in the given diagram of $K$ with either a " 0 smoothing" ()() or with a "1-smoothing" $(\asymp)$ (see Figure 2

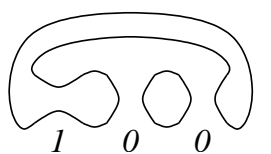
for the distinction). As our $K$ has 3 crossings, it has $2^{3}=8$ smoothings. Given the ordering on the crossings of $K$ these 8 smoothings naturally correspond to the vertices of the 3 -dimensional cube $\{0,1\}^{3}$.

2.6 Edges Each edge of the cube is labeled by a cobordism between the smoothing on the tail of that edge and the smoothing on its head - an oriented two dimensional surfaces

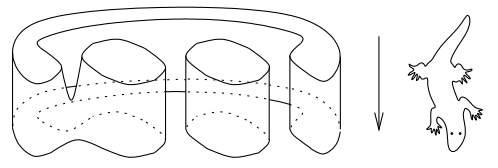
embedded in $\mathbb{R}^{2} \times[0,1]$ whose boundary lies entirely in $\mathbb{R}^{2} \times\{0,1\}$ and whose "top" boundary is the "tail" smoothing and whose "bottom" boundary is the "head" smoothing. Specifically, to get the cobordism for an edge $\left(\xi_{i}\right) \in\{0,1, \star\}^{3}$ for which $\xi_{j}=\star$ we remove a disk neighborhood of the crossing $j$ from the smoothing $\xi(0):=\left.\xi\right|_{\star \rightarrow 0}$ of $K$, cross with $[0,1]$, and fill the empty cylindrical slot around the missing crossing with a saddle cobordism . Only one such cobordism is displayed in full in Figure 1 — the one corresponding to the edge 
$\star 00$. The other 11 cobordisms are only shown in a diagrammatic form, where the diagram-piece $)($ stands for the saddle cobordism with top $)($ and bottom $\asymp$.

2.7 Signs While easy to miss at first glance, the final ingredient in Figure 1 is nevertheless significant. Some of the edge cobordisms (namely, the ones on edges $01 \star, 10 \star, 1 \star 0$ and

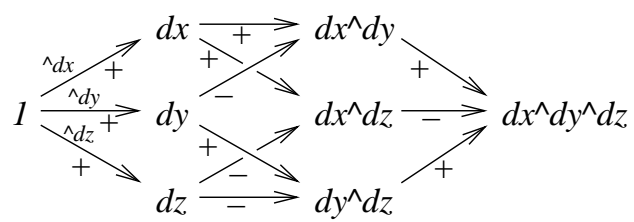
$1 \star 1)$ also carry little 'minus' $(-)$ signs. The picture on the right explains how these signs are determined from a basis of the exterior algebra in 3 (or in general, $n$ ) generators and from the exterior multiplication operation. Alternatively, if an edge $\xi$ is labeled by a sequence $\left(\xi_{i}\right)$ in the alphabet $\{0,1, \star\}$ and if $\xi_{j}=\star$, then the sign on the edge $\xi$ is $(-1)^{\xi}:=(-1)^{\sum_{i<j} \xi_{i}}$.

2.8 Tangles It should be clear to the reader how construct a picture similar to the one in Figure 1 for an arbitrary link diagram with possibly more (or less) crossings. In fact, it should also be clear how to construct such a picture for any tangle (a part of a link diagram bounded inside a disk); the main dif-
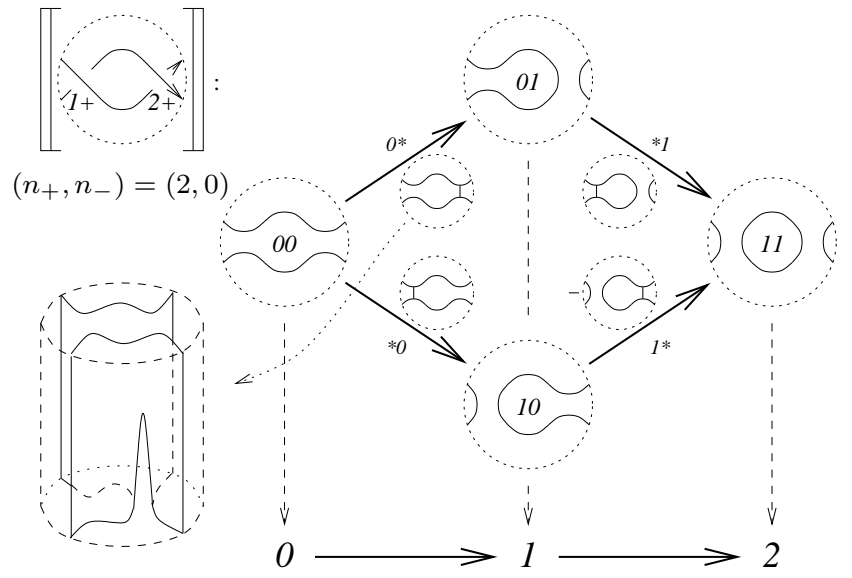
ference is that now all cobor-

disms are bounded within a cylinder, and the part of their boundary on the sides of the cylinder is a union of vertical straight lines. An example is on the right.

\section{A frame for our picture}

Following the previous section, we know how to associate an intricate but sofar-meaningless picture of a certain $n$-dimensional cube of smoothings and cobordisms to every link or tangle diagram $T$. We plan to interpret such a cube as a complex (in the sense of homological algebra), denoted [T], by thinking of all smoothings as spaces and of all cobordisms as maps. We plan to set the 
$r$ 'th chain space $\llbracket T]^{r-n_{-}}$of the complex $\llbracket T \rrbracket$ to be the "direct sum" of the $\left(\begin{array}{l}n \\ r\end{array}\right)$ "spaces" (ie, smoothings) at height $r$ in the cube and to sum the given "maps" (ie, cobordisms) to get a "differential" for $[T]$.

The problem, of course, is that smoothings aren't spaces and cobordisms aren't maps. They are, though, objects and morphisms respectively in a certain category $\mathcal{C}_{o b}{ }^{3}(\partial T)$ defined below.

Definition $3.1 \mathcal{C}_{o b}{ }^{3}(\emptyset)$ is the category whose objects are smoothings (ie, simple curves in the plane) and whose morphisms are cobordisms between such smoothings as in Section 2 6, regarded up to boundarypreserving isotopiest Likewise if $B$ is a

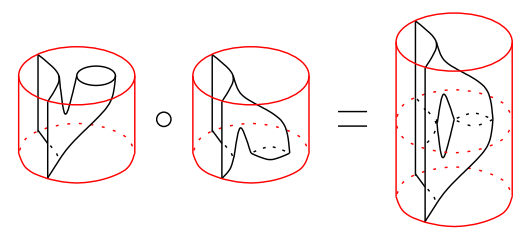
finite set of points on the circle (such as the boundary $\partial T$ of a tangle $T$ ), then $\mathcal{C} b^{3}(B)$ is the category whose objects are smoothings with boundary $B$ and whose morphisms are cobordisms between such smoothings as in Section 2,8, regarded up to boundary-preserving isotopies. In either case the composition of morphisms is given by placing one cobordism atop the other. We will use the notation $\mathcal{C}_{o} b^{3}$ as a generic reference either to $\mathcal{C}_{o b} b^{3}(\emptyset)$ or to $\mathcal{C}_{o} b^{3}(B)$ for some $B$.

Next, let us see how in certain parts of homological algebra general "objects" and "morphisms" can replace spaces and maps; ie, how arbitrary categories can replace the Abelian categories of vector spaces and/or $\mathbb{Z}$-modules which are more often used in homological algebra.

An pre-additive category is a category in which the sets of morphisms (between any two given objects) are Abelian groups and the composition maps are bilinear in the obvious sense. Let $\mathcal{C}$ be some arbitrary category. If $\mathcal{C}$ is pre-additive, we leave it untouched. If it isn't pre-additive to start with, we first make it pre-additive by extending every set of morphisms $\operatorname{Mor}\left(\mathcal{O}, \mathcal{O}^{\prime}\right)$ to also allow formal $\mathbb{Z}$-linear combinations of "original" morphisms and by extending the composition maps in the natural bilinear manner. In either case $\mathcal{C}$ is now pre-additive.

Definition 3.2 Given a pre-additive category $\mathcal{C}$ as above, the pre-additive category $\operatorname{Mat}(\mathcal{C})$ is defined as follows:

\footnotetext{
${ }^{4}$ A slightly different alternative for the choice of morphisms is mentioned in Section 11.3
} 
- The objects of $\operatorname{Mat}(\mathcal{C})$ are formal direct sums (possibly empty) $\oplus_{i=1}^{n} \mathcal{O}_{i}$ of objects $\mathcal{O}_{i}$ of $\mathcal{C}$.

- If $\mathcal{O}=\oplus_{i=1}^{m} \mathcal{O}_{i}$ and $\mathcal{O}^{\prime}=\oplus_{j=1}^{n} \mathcal{O}_{j}^{\prime}$, then a morphism $F: \mathcal{O}^{\prime} \rightarrow \mathcal{O}$ in $\operatorname{Mat}(\mathcal{C})$ will be an $m \times n$ matrix $F=\left(F_{i j}\right)$ of morphisms $F_{i j}: \mathcal{O}_{j}^{\prime} \rightarrow \mathcal{O}_{i}$ in $\mathcal{C}$.

- Morphisms in $\operatorname{Mat}(\mathcal{C})$ are added using matrix addition.

- Compositions of morphisms in $\operatorname{Mat}(\mathcal{C})$ are defined by a rule modeled on matrix multiplication, but with compositions in $\mathcal{C}$ replacing the multiplication of scalars,

$$
\left(\left(F_{i j}\right) \circ\left(G_{j k}\right)\right)_{i k}:=\sum_{j} F_{i j} \circ G_{j k} .
$$

$\operatorname{Mat}(\mathcal{C})$ is often called "the additive closure of $\mathcal{C}$ ".

It is often convenient to represent objects of $\operatorname{Mat}(\mathcal{C})$ by column vectors and morphisms by bundles 5 of arrows pointing from one column to another. With this image, the composition $(F \circ G)_{i k}$ becomes a sum over all routes from $\mathcal{O}_{k}^{\prime \prime}$ to $\mathcal{O}_{i}$ formed by connecting arrows. See Figure 3.

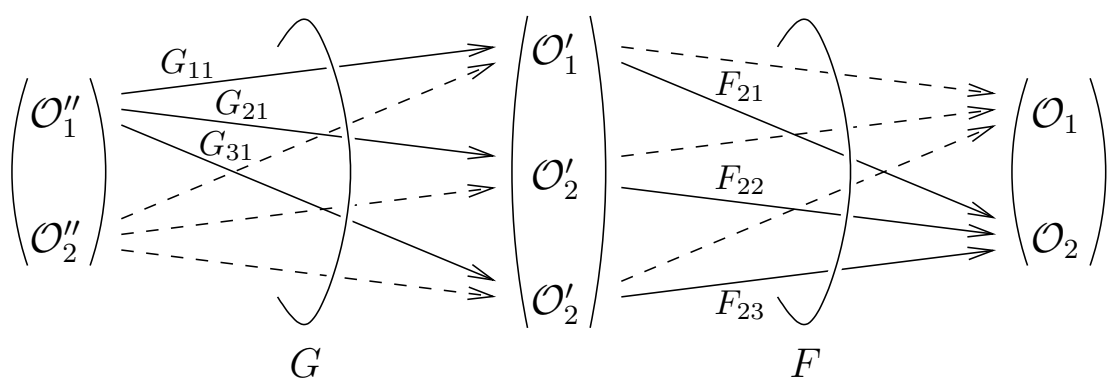

Figure 3: Matrices as bundles of morphisms, the composition $F \circ G$ and the matrix element $(F \circ G)_{21}=F_{21} \circ G_{11}+F_{22} \circ G_{21}+F_{23} \circ G_{31}$ (in solid lines).

A quick glance at Figure 1] should convince the reader that it can be interpreted as a chain of morphisms $\left[K \rrbracket=\left([K]^{-3} \longrightarrow[K]^{-2} \longrightarrow[K]^{-1} \longrightarrow[K]^{0}\right)\right.$ in $\operatorname{Mat}\left(\operatorname{Cob}^{3}\right)$, where $K={ }_{1-2-}$ (if an arrow is missing, such as between the vertices 001 and 110 of the cube, simply regard it as 0 ). Likewise, if $T$ is an $n$-crossing tangle, Section 2 tells us how it can be interpreted as a length $n$

\footnotetext{
5 "Bundle" in the non-technical sense. Merriam-Webster: bundle: "a group of things fastened together for convenient handling".
} 
chain $[T]=\left([T]^{-n_{-}} \longrightarrow[T]^{-n_{-}+1} \longrightarrow \cdots \longrightarrow[T]^{n_{+}}\right)$. (Strictly speaking, we didn't specify how to order the equal-height layers of an $n$-dimensional cube as "column vector". Pick an arbitrary such ordering.) Let us make a room for such chains by mimicking the standard definition of complexes:

Definition 3.3 Given a preadditive category $\mathcal{C}$, let $\operatorname{Kom}(\mathcal{C})$ be the category of complexes over $\mathcal{C}$, whose objects are chains

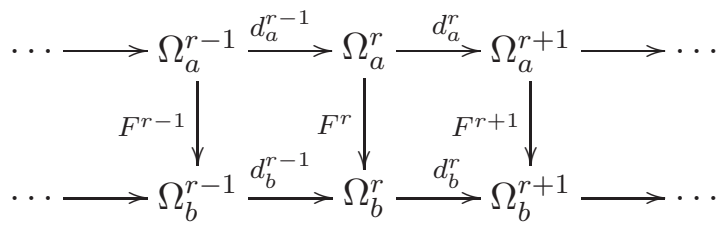

of finite length $\cdots \longrightarrow \Omega^{r-1} \stackrel{d^{r-1}}{\longrightarrow} \Omega^{r} \stackrel{d^{r}}{\longrightarrow} \Omega^{r+1} \longrightarrow \cdots$ for which the composition $d^{r} \circ d^{r-1}$ is 0 for all $r$, and whose morphisms $F:\left(\Omega_{a}^{r}, d_{a}\right) \rightarrow\left(\Omega_{b}^{r}, d_{b}\right)$ are commutative diagrams as displayed on the right, in which all arrows are morphisms in $\mathcal{C}$. Like in ordinary homological algebra, the composition $F \circ G$ in $\operatorname{Kom}(\mathcal{C})$ is defined via $(F \circ G)^{r}:=F^{r} \circ G^{r}$.

Proposition 3.4 For any tangle (or knot/link) diagram $T$ the chain $[T]$ is a complex in $\operatorname{Kom}\left(\operatorname{Mat}\left(\mathcal{C o b}^{3}(\partial T)\right)\right)$. That is, $d^{r} \circ d^{r-1}$ is always 0 for these chains.

Proof We have to show that every square face of morphisms in the cube of $T$ anticommutes. Every square face of the cube of

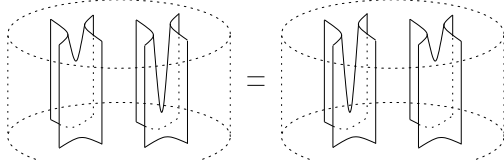
signs of Section 2, 6 carries an odd number of minus signs (this follows readily from the anti-commutativity of exterior multiplication, $\left.d x_{i} \wedge d x_{j}=-d x_{j} \wedge d x_{i}\right)$. Hence, all signs forgotten, we have to show that every square face in the cube of $T$ positively commutes. This is simply the fact that spatially separated saddles can be time-reordered within a cobordism by an isotopy.

\section{Invariance}

\subsection{Preliminaries}

The "formal" complex $[T \rrbracket$ is not a tangle invariant in any sense. We will claim and prove, however, that $[T]$, regarded within $\operatorname{Kom}\left(\operatorname{Mat}\left(\mathcal{C}_{o b}{ }^{3}\right)\right)$ for some quotient $\mathcal{C o b}_{/ l}^{3}$ of $\mathcal{C o b}^{3}$, is invariant up to homotopy. But first we have to define these terms. 


\subsubsection{Homotopy in formal complexes}

Let $\mathcal{C}$ be a category. Just like in ordinary homological algebra, we say that two morphisms $F, G:\left(\Omega_{a}^{r}\right) \rightarrow\left(\Omega_{b}^{r}\right)$ in $\operatorname{Kom}(\mathcal{C})$

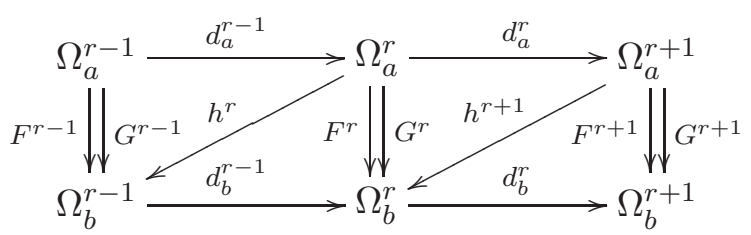
are homotopic (and we write $F \sim G)$ if there exists "backwards diagonal" morphisms $h^{r}: \Omega_{a}^{r} \rightarrow \Omega_{b}^{r-1}$ so that $F^{r}-G^{r}=h^{r+1} d^{r}+d^{r-1} h^{r}$ for all $r$.

Many of the usual properties of homotopies remain true in the formal case, with essentially the same proofs. In particular, homotopy is an equivalence relation and it is invariant under composition both on the left and on the right; if $F$ and $G$ are homotopic and $H$ is some third morphism in $\operatorname{Kom}(\mathcal{C})$, then $F \circ H$ is homotopic to $G \circ H$ and $H \circ F$ is homotopic to $H \circ G$, whenever these compositions make sense. Thus we can make the following definition:

Definition $4.1 \operatorname{Kom}_{/ h}(\mathcal{C})$ is $\operatorname{Kom}(\mathcal{C})$ modulo homotopies. That is, $\operatorname{Kom}_{/ h}(\mathcal{C})$ has the same objects as $\operatorname{Kom}(\mathcal{C})$ (formal complexes), but homotopic morphisms in $\operatorname{Kom}(\mathcal{C})$ are declared to be the same in $\operatorname{Kom}_{/ h}(\mathcal{C})$ (the $/ h$ stands for "modulo homotopy").

As usual, we say that two complexes $\left(\Omega_{a}^{r}\right)$ and $\left(\Omega_{b}^{r}\right)$ in $\operatorname{Kom}(\mathcal{C})$ are homotopy equivalent (and we write $\left.\left(\Omega_{a}^{r}\right) \sim\left(\Omega_{b}^{r}\right)\right)$ if they are isomorphic in $\mathrm{Kom}_{/ h}(\mathcal{C})$. That is, if there are morphisms $F:\left(\Omega_{a}^{r}\right) \rightarrow\left(\Omega_{b}^{r}\right)$ and $G:\left(\Omega_{b}^{r}\right) \rightarrow\left(\Omega_{a}^{r}\right)$ so that the compositions $G \circ F$ and $F \circ G$ are homotopic to the identity automorphisms of $\left(\Omega_{a}^{r}\right)$ and $\left(\Omega_{b}^{r}\right)$, respectively. It is routine to verify that homotopy equivalence is an equivalence relation on complexes.

\subsubsection{The quotient $\mathcal{C o b}_{/ l}^{3}$ of $\mathcal{C}_{o b}{ }^{3}$}

We mod out the morphisms of the category $\mathcal{C}_{0} b^{3}$ by the relations $S, T$ and $4 T u$ defined below and call the resulting quotient $\mathcal{C}_{o b}^{3}{ }_{/ l}$ (the $/ l$ stands for "modulo local relations").

The $S$ relation says that whenever a cobordism contains a connected component which is a closed sphere (with no boundary), it is set equal to zero (remember that we make all categories pre-

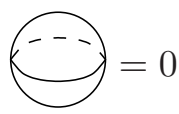
additive, so 0 always makes sense). 
The $T$ relation says that whenever a cobordism contains a connected component which is a closed torus (with no boundary), that component may be dropped and replaced by a

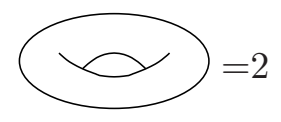
numerical factor of 2 (remember that we make all categories pre-additive, so multiplying a cobordism by a numerical factor makes sense).

To understand $4 \mathrm{Tu}$, start from some given cobordism $C$ and assume its intersection with a certain ball is the union of four disks

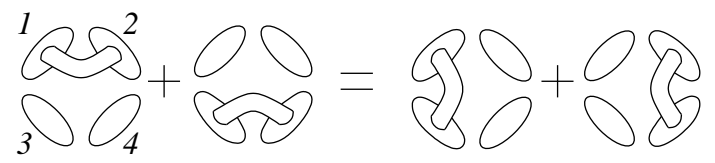
$D_{1}$ through $D_{4}$ (these disks may well be on different connected components of $C)$. Let $C_{i j}$ denote the result of removing $D_{i}$ and $D_{j}$ from $C$ and replacing them by a tube that has the same boundary. The "four tube" relation $4 T u$ asserts that $C_{12}+C_{34}=C_{13}+C_{24}$.

The local nature of the $S, T$ and $4 T u$ relations implies that the composition operations remain well defined in $\mathcal{C}_{0} b_{/ l}^{3}$ and hence it is also a pre-additive category.

\subsection{Statement}

Throughout this paper we will often short $\operatorname{Kob}(\emptyset), \operatorname{Kob}(B)$ and $\operatorname{Kob}$ for $\operatorname{Kom}\left(\operatorname{Mat}\left(\mathcal{C o b}_{/ l}^{3}(\emptyset)\right)\right), \operatorname{Kom}\left(\operatorname{Mat}\left(\mathcal{C o b}_{/ l}^{3}(B)\right)\right)$ and $\operatorname{Kom}\left(\operatorname{Mat}\left(\mathcal{C}_{o} b_{l l}^{3}\right)\right)$. Likewise

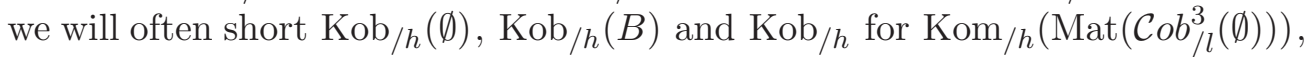

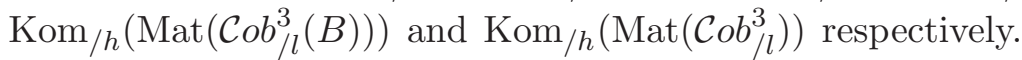

Theorem 1 (The Invariance Theorem) The isomorphism class of the complex $[T]$ regarded in $\mathrm{Kob}_{/ h}$ is an invariant of the tangle $T$. That is, it does not depend on the ordering of the layers of a cube as column vectors and on the ordering of the crossings and it is invariant under the three Reidemeister moves (reproduced in Figure 4).
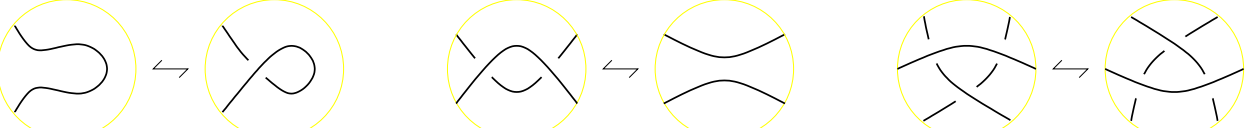

Figure 4: The three Reidemeister moves $R 1, R 2$ and $R 3$. 


\subsection{Proof}

The independence on the ordering of the layers of a cube as column vectors is left as an exercise to the reader with no hints supplied. The independence on the ordering of the crossings is also left as an exercise to the reader (hint: when reordering, take signs from the signs that appear in the usual action of a symmetric group on the basis of an exterior algebra). Invariance under the Reidemeister moves is shown, in this section, just for the "local" tangles representing these moves. Invariance under the Reidemeister moves applied within larger tangles or knots or links follows from the nearly tautological good behaviour of [T] with respect to tangle compositions discussed in Section [5 (see also Exercise 4.8).

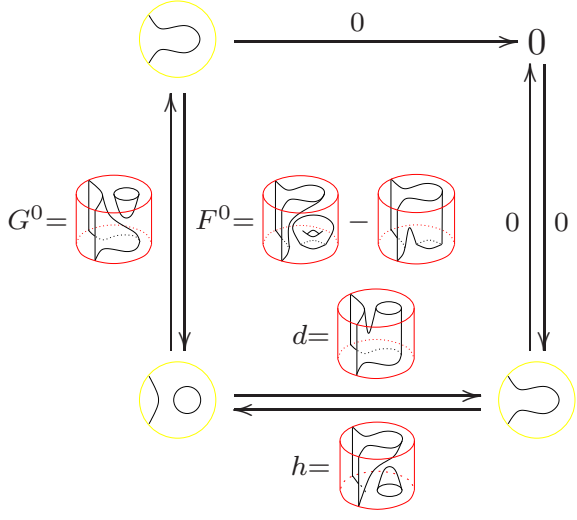

Figure 5: Invariance under $R 1$

Invariance under the Reidemeister move $R 1$ (See Figure 5) We have to show that the formal complex $\llbracket \supset \|=(0 \longrightarrow つ \longrightarrow 0)$ is homotopy equivalent to the formal complex $\llbracket \curvearrowright \|=(0 \longrightarrow \underline{>} \stackrel{d}{\longrightarrow} \supset \longrightarrow 0)$, in which $d=f$ (in both complexes we have underlined the 0th term). To do this we construct (homotopically inverse) morphisms $F: \llbracket \supset \rrbracket \rightarrow \llbracket \triangleright \rrbracket$ and $G: \llbracket \triangleright \rrbracket \rightarrow \llbracket \supset \rrbracket$. The morphism $F$ is defined by $F^{0}=$ (in words: a vertical curtain union a torus with a downward-facing disk removed, minus a simple saddle) and $F^{\neq 0}=0$. The morphism $G$ is defined by $G^{0}=$ 
(a vertical curtain union a cup) and $G^{\neq 0}=0$. The only non-trivial commutativity to verify is $d F^{0}=0$, which follows from 490 (f), and where the latter identity holds because both of its sides are the same - vertical curtains with an extra handle attached. If follows from the $T$ relation that $G F=I$.

Finally, consider the (homotopy) morphism $h=\sqrt{\theta}: \llbracket \triangleright \|^{1}=$ $\supset \rightarrow \rtimes=\llbracket \triangleright \rrbracket^{0}$. Clearly, $F^{1} G^{1}-I+d h=-I+d h=0$. We claim that it follows from the $4 T u$ relation that $F^{0} G^{0}-I+h d=0$ and hence $F G \sim I$ and we have proven that $\llbracket \supset \rrbracket \sim \llbracket \triangleright \rrbracket$.

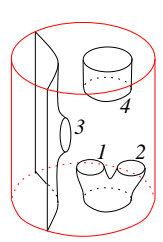
Indeed, let $C$ be the cobordism (with four punctures labeled 1-4) shown on the right, and consider the cobordisms $C_{i j}$ constructed from it as in Section 4.1.2 Then $C_{12}$ and $C_{13}$ are the first and second summands in $F^{0} G^{0}, C_{24}$ is the identity morphisms $I$ and $C_{34}$ is $h d$. Hence the $4 T u$ relation $C_{12}-C_{13}-C_{24}+C_{34}=0$ is precisely our assertion, $F^{0} G^{0}-I+h d=0$.

Invariance under the Reidemeister move $R 2$ This invariance proof is very similar in spirit to the previous one, and hence we will allow ourselves to be brief. The proof appears in whole in Figure 6 let us just add some explanatory words. In that figure the top row is the formal complex $\llbracket \rrbracket$ and the bottom row is the formal complex [®]. Also, all eastward arrows are (components of) differentials, the southward arrows are the components of a morphism $F: \llbracket \rrbracket \rightarrow \llbracket ®$, the northward arrows are the components of a morphism $G: \llbracket \bowtie \rrbracket \llbracket \rrbracket$, and the westward arrows are the non-zero components of a homotopy $h$ proving that $F G \sim I$. Finally, in Figure 6 the symbol $)$ ( (or its variants, $\nearrow$, etc.) stands for the saddle cobordism $)(:)(\longrightarrow \asymp$ (or $\Upsilon: \asymp \rightarrow)$ (, etc.) as in Figure 1, and the symbols $\mathscr{Q}$ and $\bigcirc$ stand for the cap morphism $\propto: \emptyset \rightarrow \bigcirc$ and the cup morphism $Q: \bigcirc \rightarrow \emptyset$.

We leave it for the reader to verify the following facts which together constitute a proof of invariance under the Reidemeister move $R 2$ :

- $d F=0$ (only uses isotopies).

- $G d=0$ (only uses isotopies).

- $G F=I$ (uses the relation $S$ ).

- The hardest $-F G-I=h d+d h$ (uses the $4 T u$ relation). 


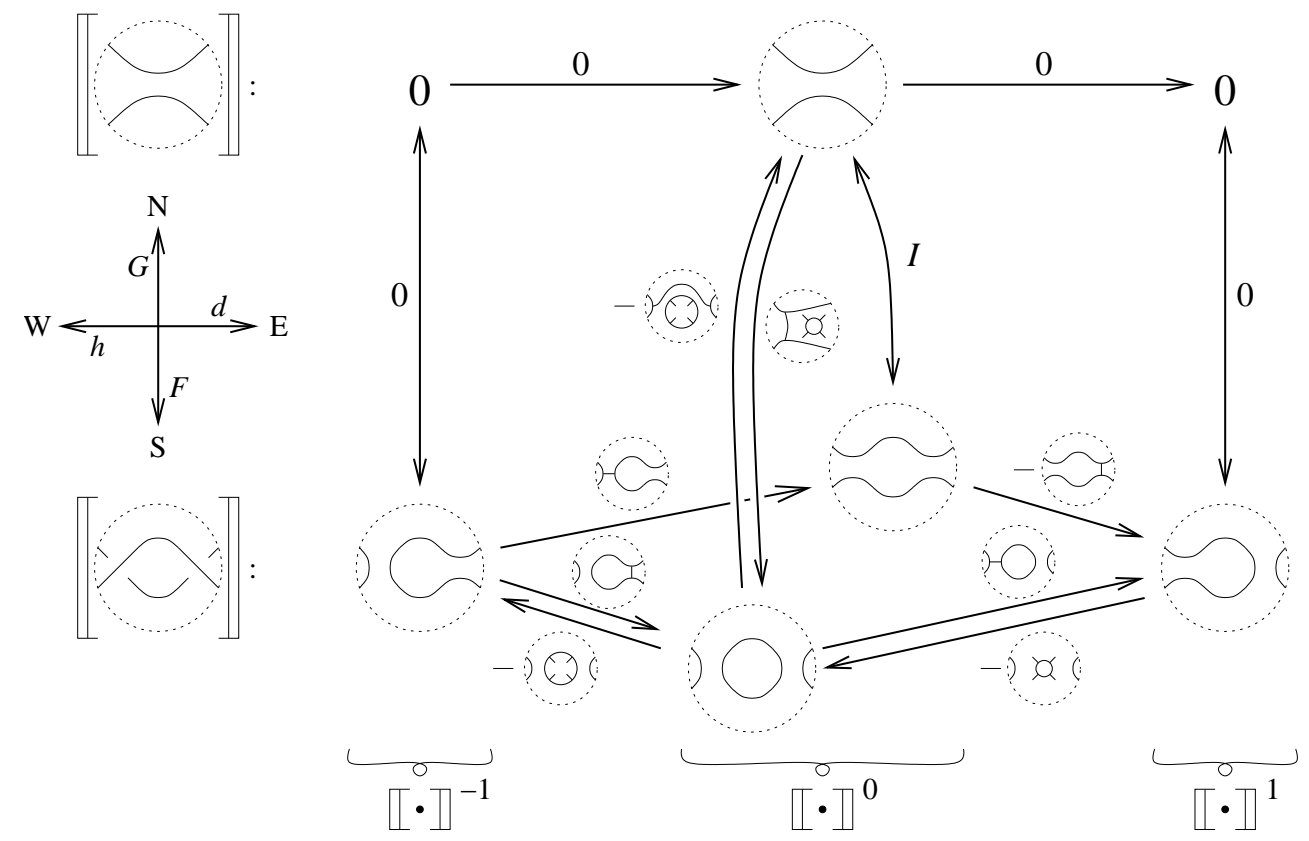

Figure 6: Invariance under the Reidemeister move $R 2$.

Remark 4.2 The morphism $G: \llbracket \otimes \rrbracket \rightarrow \llbracket \backsim \rrbracket$ at the heart of the above proof is a little more than a homotopy equivalence. A routine check shows that it is in fact a strong deformation retract in the sense of the following definition.

Definition 4.3 A morphism of complexes $G: \Omega_{a} \rightarrow$ $\Omega_{b}$ is said to be a strong deformation retract if there is a morphism $F: \Omega_{b} \rightarrow \Omega_{a}$ and homotopy maps $h$ from $\Omega_{a}$ to itself so that $G F=I, I-F G=d h+h d$ and $h F=0$. In this case we say that $F$ is the $i n$ clusion in a strong deformation retract. Note that a strong deformation retract is in particular a homotopy equivalence. The geometric origin of this notion

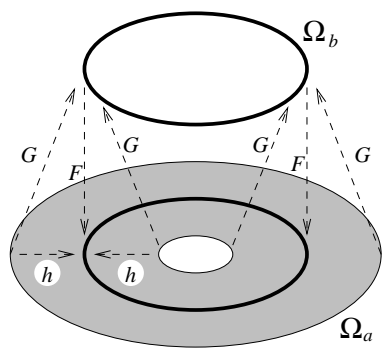
is the standard notion of a strong deformation retract in homotopy theory as sketched on the right.

Invariance under the Reidemeister move $R 3$ This is the easiest and hardest move. Easiest because it doesn't require any further use of the $S, T$ and 
$4 T u$ relations - it just follows from the $R 2$ move and some 'soft' algebra (just as in the case of the Kauffman bracket, whose invariance under $R 3$ follows 'for free' from its invariance under $R 2$; see eg, 9, Lemma 2.4]). Hardest because it involves the most crossings and hence the most complicated complexes. We will attempt to bypass that complexity by appealing to some standard constructions and results from homological algebra.

Let $\Psi:\left(\Omega_{0}^{r}, d_{0}\right) \rightarrow\left(\Omega_{1}^{r}, d_{1}\right)$ be a morphism of complexes. The cone $\Gamma(\Psi)$ of $\Psi$ is the complex with chain spaces $\Gamma^{r}(\Psi)=\Omega_{0}^{r+1} \oplus \Omega_{1}^{r}$ and with differentials $\tilde{d}^{r}=\left(\begin{array}{cc}-d_{0}^{r+1} & 0 \\ \Psi^{r+1} & d_{1}^{r}\end{array}\right)$. The follow-

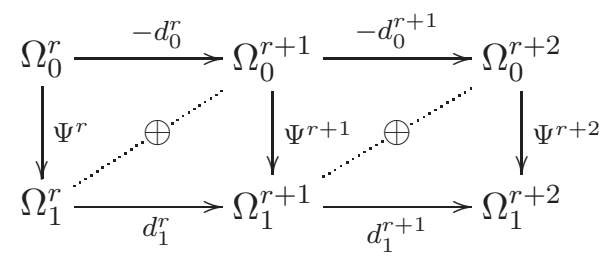
ing two lemmas explain the relevance of cones to the task at hand and are easy 6 to verify:

Lemma $4.4 \llbracket \times \rrbracket=\Gamma(\llbracket)-(\rrbracket)[-1]$ and $\llbracket \lambda \rrbracket=\Gamma(\llbracket Y])$, where $\llbracket r-(\rrbracket$ and $\llbracket Y \rrbracket$ are the saddle morphisms $\llbracket \mathcal{L}-(-\rrbracket: \llbracket)(\rrbracket \rightarrow \llbracket \smile \rrbracket$ and $\llbracket \backslash \rrbracket: \llbracket \asymp \rrbracket \rightarrow \llbracket)(\rrbracket$ and where . $[s]$ is the operator that shifts complexes $s$ units to the left: $\Omega[s]^{r}:=\Omega^{r+s}$.
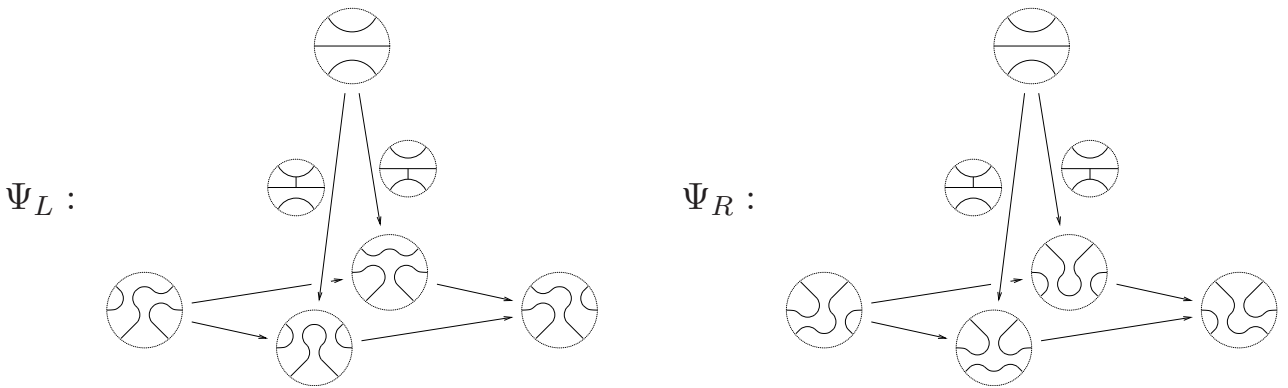

Figure 7: The two sides of the Reidemeister move $R 3$.

Lemma 4.5 The cone construction is invariant up to homotopy under compositions with the inclusions in strong deformation retracts. That is, consider the diagram of complexes and morphisms that appears on the right. If in that diagram $G_{0}$ is a strong deformation retract with inclusion $F_{0}$, then

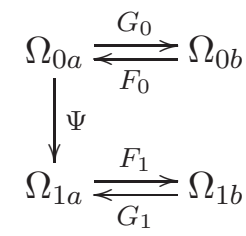

${ }^{6} \mathrm{Hard}$, if one is punctual about signs... . 
the cones $\Gamma(\Psi)$ and $\Gamma\left(\Psi F_{0}\right)$ are homotopy equivalent, and if $G_{1}$ is a strong deformation retract with inclusion $F_{1}$, then the cones $\Gamma(\Psi)$ and $\Gamma\left(F_{1} \Psi\right)$ are homotopy equivalent. (This lemma remains true if $F_{0,1}$ are strong deformation retracts and $G_{0,1}$ are the corresponding inclusions, but we don't need that here).

We note that Lemma 4.4 can also be interpreted (and remains true) in a "skein theoretic" sense, where each of $\lambda$ and $\boldsymbol{H}$ (or $\lambda$ and $\nearrow$ ) represents just a small disk neighborhood inside an otherwise-equal bigger tangle. Thus, applying Lemma 4.4 to the bottom crossing in the tangle we find that the complex

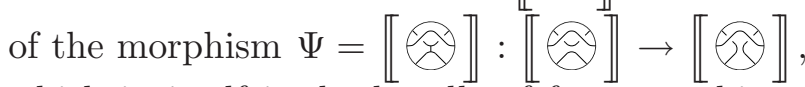

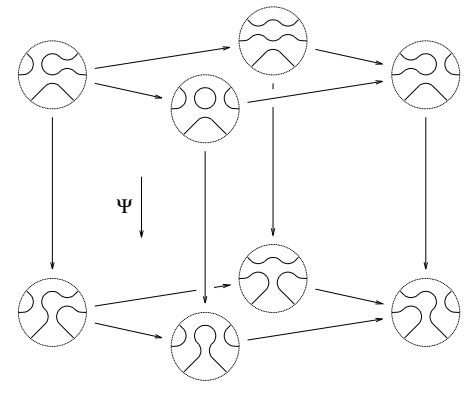
which in itself is the bundle of four morphisms corresponding to the four smoothings of the two remaining crossings of (see the diagram on the right). We can now use Lemma 4.5 and the inclusion $F$ of Figure 6 (notice Remark 4.2) to replace the top layer of this cube by a

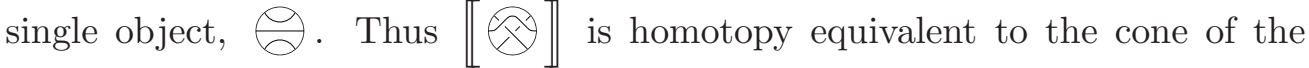
vertical morphism $\Psi_{L}=\Psi F$ of Figure 7 A similar treatment applied to the complex $\llbracket \rrbracket$ yields the cone of the morphism $\Psi_{R}$ of Figure 7. But up to isotopies $\Psi_{L}$ and $\Psi_{R}$ are the same.

Given that we distinguish left from right, there is another variant of the third Reidemeister move to check that invariance here can be proven in a similar way, except using the second half of Lemma 4.5.

Proof of Lemma 4.5 Let $h_{0}: \Omega_{0 a}^{\star} \rightarrow \Omega_{0 a}^{\star-1}$ be a homotopy for which $I-$ $F_{0} G_{0}=d h_{0}+h_{0} d$ and $h_{0} F_{0}=0$. Then the diagram in Figure 8 defines morphisms $\Gamma\left(\Psi F_{0}\right) \underset{\tilde{G}_{0}}{\stackrel{\tilde{F}_{0}}{\rightleftarrows}} \Gamma(\Psi)$ and a homotopy $\tilde{h}_{0}: \Gamma(\Psi)^{\star} \rightarrow \Gamma(\Psi)^{\star-1}$. We leave it to the reader to verify that $\tilde{F}_{0}$ and $\tilde{G}_{0}$ are indeed morphisms of complexes and that $\tilde{G}_{0} \tilde{F}_{0}=I$ and $I-\tilde{F}_{0} \tilde{G}_{0}=\tilde{d} \tilde{h}_{0}+\tilde{h}_{0} \tilde{d}$ and hence $\Gamma\left(\Psi F_{0}\right)$ and $\Gamma(\Psi)$ are homotopy equivalent. A similar argument shows that $\Gamma(\Psi)$ and $\Gamma\left(F_{1} \Psi\right)$ are also homotopy equivalent. 


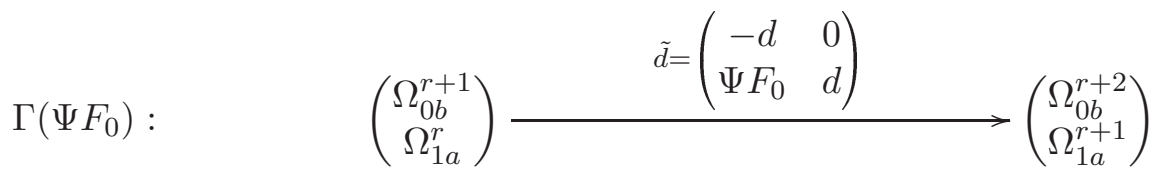

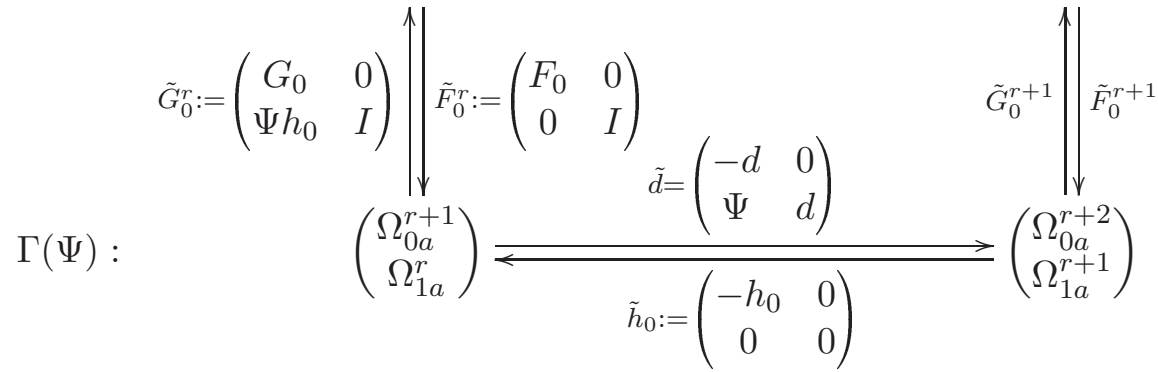

Figure 8

Remark 4.6 The proof of invariance under the Reidemeister move $R 3$ was presented in a slightly roundabout way, using cones and their behaviour under retracts. But there is no difficulty in unraveling everything to get concrete (homotopically invertible) morphisms between the formal complexes at the two sides of $R 3$. This is done (for just one of the two variants of $R 3$ ) in Figure 9 The most interesting cobordism in Figure 9 is displayed - a cubic saddle 7 $\left(z, 3 \operatorname{Re}\left(z^{3}\right)\right)$ bound in the cylinder $[z \leq 1] \times[-1,1]$ plus a cup and a cap.

Exercise 4.7 Verify that Figure 9 indeed defines a map between complexes that the morphism defined by the downward arrows commutes with the (right pointing) differentials.

Exercise 4.8 Even though this will be done in a formal manner in the next section, we recommend that the reader will pause here to convince herself that the "local" proofs above generalize to Reidemeister moves performed within larger tangles, and hence that Theorem 1 is verified. The mental picture you will thus create in your mind will likely be a higher form of understanding than its somewhat arbitrary serialization into a formal stream of words below.

\footnotetext{
${ }^{7}$ A "monkey saddle", comfortably seating a monkey with two legs and a tail.
} 


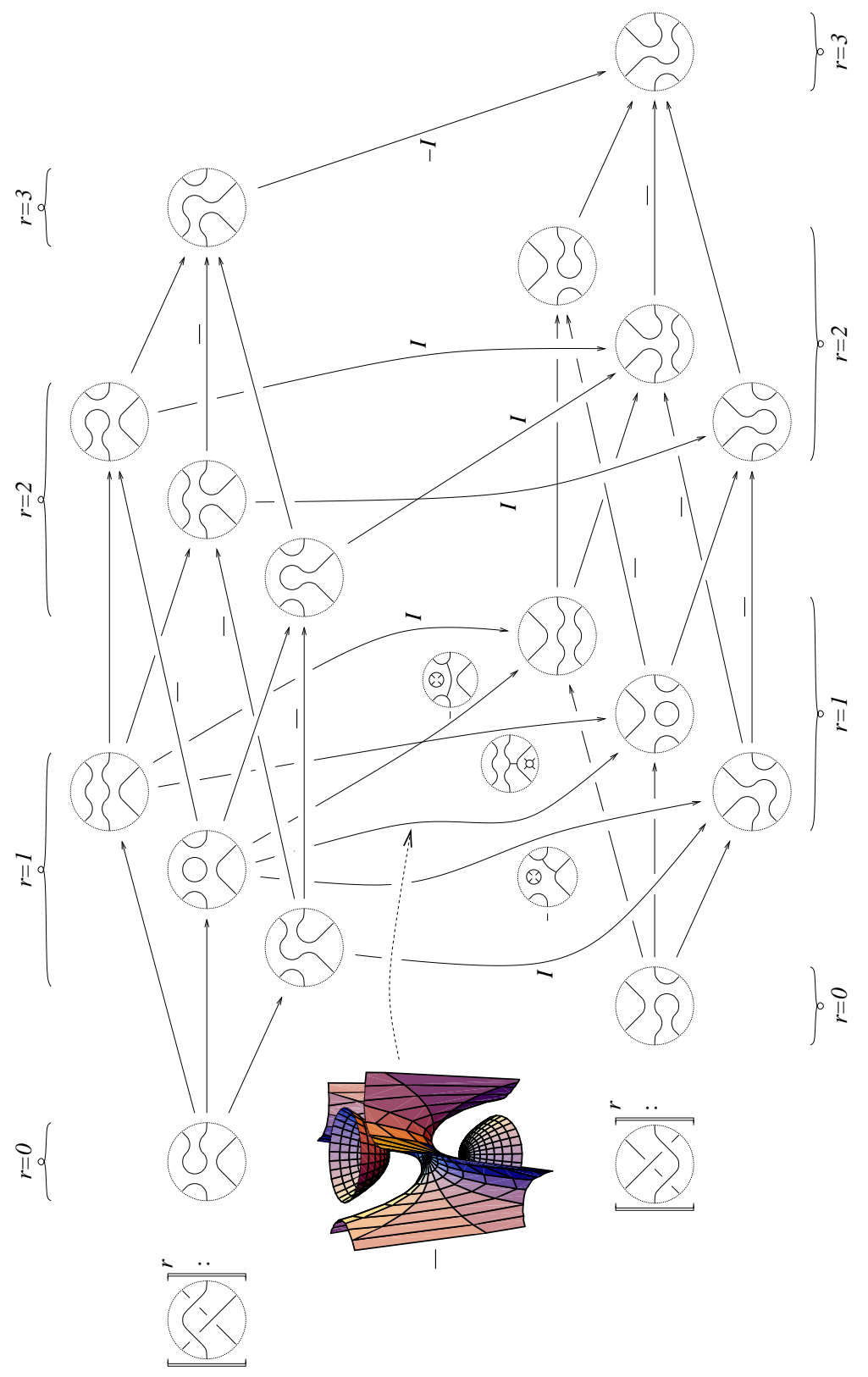

Figure 9: Invariance under $R 3$ in more detail than is strictly necessary. Notice the minus signs and consider all missing arrows between the top layer and the bottom layer as 0 . 


\section{$5 \quad$ Planar algebras and tangle compositions}

Overview Tangles can be composed in a variety of ways. Indeed, any $d$-input "planar arc diagram" $D$ (such as the 4-input example on the right) yields an operator taking $d$ tangles as inputs and producing a single "bigger" tangle as an output by placing the $d$ input tangles into the $d$ holes of $D$. The purpose of this section is to define precisely what "planar arc diagrams" are and explain how they turn the collection of tangles into a "planar algebra", to explain how

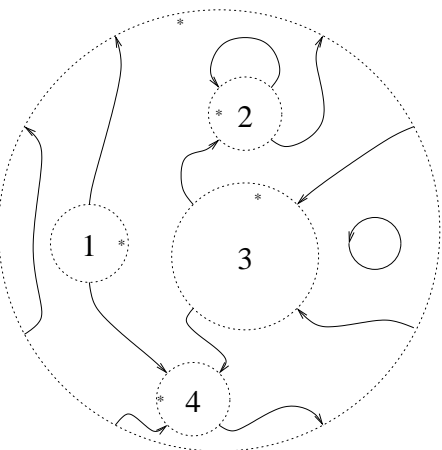
formal complexes in Kob also form a planar algebra and to note that the Khovanov bracket [.] is a planar algebra morphism from the planar algebra of tangles to the planar algebra of such complexes. Thus Khovanov brackets "compose well". In particular, the invariance proofs of the previous section, carried out at the local level, lift to global invariance under Reidemeister moves.

Definition 5.1 A $d$-input planar arc diagram $D$ is a big "output" disk with $d$ smaller "input" disks removed, along with a collection of disjoint embedded oriented arcs that are either closed or begin and end on the boundary. The input disks are numbered 1 through $d$, and there is a base point $(*)$ marked on each of the input disks as well as on the output disk. Finally, this information is considered only up to planar isotopy. An unoriented planar arc diagram is the same, except the orientation of the arcs is forgotten.

Definition 5.2 Let $\mathcal{T}^{0}(k)$ denote the collection of all $k-$ ended unoriented tangle diagrams (unoriented tangle diagrams in a disk, with $k$ ends on the boundary of the disk)

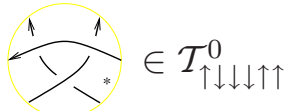
in a based disk (a disk with a base point marked on its boundary). Likewise, if $s$ is a string of in $(\uparrow)$ and out $(\downarrow)$ symbols with a total length of $|s|$, let $\mathcal{T}^{0}(s)$ denote the collection of all $|s|$-ended oriented tangle diagrams in a based disk with incoming/outgoing strands as specified by $s$, starting at the base point and going around counterclockwise. Let $\mathcal{T}(k)$ and $\mathcal{T}(s)$ denote the respective quotient of $\mathcal{T}^{0}(k)$ and $\mathcal{T}^{0}(s)$ by the three Reidemeister moves (so those are spaces of tangles rather than tangle diagrams).

Clearly every $d$-input unoriented planar arc diagram $D$ defines operations (denoted by the same symbol)

$$
D: \mathcal{T}^{0}\left(k_{1}\right) \times \cdots \times \mathcal{T}^{0}\left(k_{d}\right) \rightarrow \mathcal{T}^{0}(k) \quad \text { and } \quad D: \mathcal{T}\left(k_{1}\right) \times \cdots \times \mathcal{T}\left(k_{d}\right) \rightarrow \mathcal{T}(k)
$$


by placing the $d$ input tangles or tangle diagrams into the $d$ holes of $D$ (here $k_{i}$ are the numbers of $\operatorname{arcs}$ in $D$ that end on the $i$ 'th input disk and $k$ is the number of arcs that end on the output disk). Likewise, if $D$ is oriented and $s_{i}$ and $s$ are the in/out strings read along the inputs and output of $D$ in the natural manner, then $D$ defines operations

$$
D: \mathcal{T}^{0}\left(s_{1}\right) \times \cdots \times \mathcal{T}^{0}\left(s_{d}\right) \rightarrow \mathcal{T}^{0}(s) \quad \text { and } \quad D: \mathcal{T}\left(s_{1}\right) \times \cdots \times \mathcal{T}\left(s_{d}\right) \rightarrow \mathcal{T}(s)
$$

These operations contain the identity operations on $\mathcal{T}^{(0)}(k$ or $s)$ (take "radial" $D$ of the form ) and are compatible with each other ("associative") in a natural way. In brief, if $D_{i}$ is the result of placing $D^{\prime}$ into the $i$ th hole of $D$ (provided the relevant $k / s$ match) then as operations, $D_{i}=D \circ\left(I \times \cdots \times D^{\prime} \times\right.$ $\cdots \times I)$.

In the spirit of Jones [8] we call a collection of sets $\mathcal{P}(k)($ or $\mathcal{P}(s))$ along with operations $D$ defined for each unoriented planar arc diagram (oriented planar arc diagram) a planar algebra (an oriented planar algebra), provided the radial $D$ 's act as identities and provided associativity conditions as above hold. Thus as first examples of planar algebras (oriented or not) we can take $\mathcal{T}^{(0)}(k$ or $s)$.

Another example of a planar algebra (unoriented) is the full collection $\operatorname{Obj}\left(\mathcal{C o b}_{/ l}^{3}\right)$ of objects of the category $\mathcal{C o b}_{/ l}^{3}-$ this is in fact the "flat" (no crossings) sub planar algebra of $(\mathcal{T}(k))$. An even more interesting example is the full collection $\operatorname{Mor}\left(\mathcal{C}_{0} b_{/ l}^{3}\right)$ of morphisms of $\mathcal{C} o b_{/ l}^{3}$ — indeed, if $D$ is

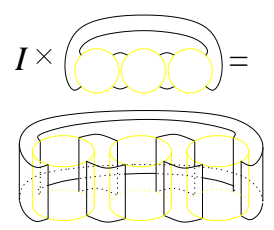
a $d$-input unoriented planar arc diagram then $D \times[0,1]$ is a vertical cylinder with $d$ vertical cylindrical holes and with vertical curtains connecting those. One can place $d$ morphisms of $\mathcal{C}_{o b} b_{l}^{3}$ (cobordisms) inside the cylindrical holes and thus get an operation $D:\left(\operatorname{Mor}\left({\mathcal{C} o b^{3}}_{l l}^{3}\right)\right)^{d} \rightarrow \operatorname{Mor}\left(\mathcal{C o b}_{/ l}^{3}\right)$. Thus $\operatorname{Mor}\left(\mathcal{C o b}_{/ l}^{3}\right)$ is also a planar algebra.

A morphism $\Phi$ of planar algebras (oriented or not) $\left(\mathcal{P}^{a}(k)\right)$ and $\left(\mathcal{P}^{b}(k)\right)$ (or $\left(\mathcal{P}^{a}(s)\right)$ and $\left.\left(\mathcal{P}^{b}(s)\right)\right)$ is a collection of maps (all denoted by the same symbol) $\Phi: \mathcal{P}^{a}(k$ or $s) \rightarrow \mathcal{P}^{b}(k$ or $s)$ satisfying $\Phi \circ D=D \circ(\Phi \times \cdots \times \Phi)$ for every $D$.

We note that every unoriented planar algebra can also be regarded as an oriented one by setting $\mathcal{P}(s):=\mathcal{P}(|s|)$ for every $s$ and by otherwise ignoring all orientations on planar arc diagrams $D$.

For any natural number $k$ let $\operatorname{Kob}(k):=\operatorname{Kom}\left(\operatorname{Mat}\left(\mathcal{C o b}_{/ l}^{3}\left(B_{k}\right)\right)\right)$ and likewise let $\mathrm{Kob}_{/ h}(k):=\operatorname{Kom}_{/ h}\left(\operatorname{Mat}\left(\mathcal{C o b}_{/ l}^{3}\left(B_{k}\right)\right)\right)$ where $B_{k}$ is some placement of $k$ points along a based circle. 


\section{Theorem 2}

(1) The collection $(\operatorname{Kob}(k))$ has a natural structure of a planar algebra.

(2) The operations $D$ on $(\operatorname{Kob}(k))$ send homotopy equivalent complexes to homotopy equivalent complexes and hence the collection $\left(\mathrm{Kob}_{/ h}(k)\right)$ also has a natural structure of a planar algebra..

(3) The Khovanov bracket [.] descends to an oriented planar algebra morphism [·] : $(\mathcal{T}(s)) \rightarrow\left(\mathrm{Kob}_{/ h}(s)\right)$.

We note that this theorem along with the results of the previous section complete the proof of Theorem 1 .

Abbreviated Proof of Theorem 2 The key point is to think of the operations $D$ as (multiple) "tensor products", thus defining these operations on Kob in analogy with the standard way of taking the (multiple) tensor product of a number of complexes.

Start by endowing $\operatorname{Obj}\left(\operatorname{Mat}\left(\mathcal{C}_{o b}^{3}{ }^{3}\right)\right)$ and $\operatorname{Mor}\left(\operatorname{Mat}\left(\mathcal{C}_{o} b_{/ l}^{3}\right)\right)$ with a planar algebra structure by extending the planar algebra structure of $\operatorname{Obj}\left(\mathcal{C o b}_{/ l}^{3}\right)$ and of $\operatorname{Mor}\left(\mathcal{C}_{o b}{ }^{3} l\right)$ in the obvious multilinear manner. Now if $D$ is a $d$-input planar arc diagram with $k_{i}$ arcs ending on the $i$ 's input disk and $k$ arcs ending on the outer boundary, and if $\left(\Omega_{i}, d_{i}\right) \in \operatorname{Kob}\left(k_{i}\right)$ are complexes, define the complex $(\Omega, d)=D\left(\Omega_{1}, \ldots, \Omega_{d}\right)$ by

$$
\begin{gathered}
\Omega^{r}:=\bigoplus_{r=r_{1}+\cdots+r_{d}} D\left(\Omega_{1}^{r_{1}}, \ldots, \Omega_{d}^{r_{d}}\right) \\
\left.d\right|_{D\left(\Omega_{1}^{r_{1}}, \ldots, \Omega_{d}^{r_{d}}\right)}:=\sum_{i=1}^{d}(-1)^{\sum_{j<i} r_{j}} D\left(I_{\Omega_{1}^{r_{1}}}, \ldots, d_{i}, \ldots, I_{\Omega_{d}^{r_{d}}}\right) .
\end{gathered}
$$

With the definition of $D\left(\Omega_{1}, \ldots, \Omega_{d}\right)$ so similar to the standard definition of a tensor product of complexes, our reader should have no difficulty verifying that the basic properties of tensor products of complexes transfer to our context. Thus a morphism $\Psi_{i}: \Omega_{i a} \rightarrow \Omega_{i b}$ induces a morphism $D\left(I, \ldots, \Psi_{i}, \ldots, I\right)$ : $D\left(\Omega_{1}, \ldots, \Omega_{i a}, \ldots, \Omega_{d}\right) \rightarrow D\left(\Omega_{1}, \ldots, \Omega_{i b}, \ldots, \Omega_{d}\right)$ and homotopies at the level of the tensor factors induce homotopies at the levels of tensor products. This concludes our abbreviated proof of parts (1) and (2) of Theorem 2 
Let $T$ be a tangle diagram with $d$ crossings, let $D$ be the $d-$ input planar arc diagram obtained from $T$ by deleting a disk neighborhood of each crossing of $T$, let $X_{i}$ be the $d$ crossings of $T$, so that each $X_{i}$ is either an $\Sigma$ or an $\lambda$ (possibly rotated). Let $\Omega_{i}$ be the complexes $\left[X_{i}\right]$; so that each $\Omega_{i}$

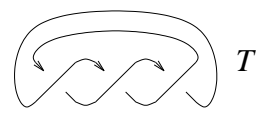
is either $\llbracket x \rrbracket=(\underline{x} \stackrel{x}{\longrightarrow} \asymp)$ or $\llbracket \lambda\rceil=(\asymp \stackrel{\Upsilon}{\longrightarrow} \underline{x})$

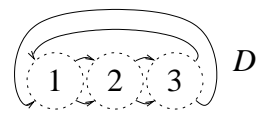
(possibly rotated, and we've underlined the 0 'th term in each complex). A quick inspection of the definition of [T] (ie, of Figure 1) and of Equation (3) shows that

$$
\left[D\left(X_{1}, \ldots, X_{d}\right)\right]=[T]=D\left(\Omega_{1}, \ldots, \Omega_{d}\right)=D\left(\left[X_{1}\right], \ldots,\left[X_{d}\right]\right) .
$$

This proves part (3) of Theorem 2 in the restricted case where all inputs are single crossings. The general case follows from this case and the associativity of the planar algebras involved.

\section{Grading and a minor refinement}

In this short section we introduce gradings into the picture, leading to a refinement of Theorem 1. While there isn't any real additional difficulty in the statement or proof of the refinement (Theorem 3 below), the benefits are great - the gradings allow us to relate [.] to the Jones polynomial (Sections 7 and [10) and allow us to easily prove the invariance of the extension of [.] to 4-dimensional cobordisms (Section 8).

Definition 6.1 A graded category is a pre-additive category $\mathcal{C}$ with the following two additional properties:

(1) For any two objects $\mathcal{O}_{1,2}$ in $\mathcal{C}$, the morphisms $\operatorname{Mor}\left(\mathcal{O}_{1}, \mathcal{O}_{2}\right)$ form a graded Abelian group, the composition maps respect the gradings (ie, $\operatorname{deg} f \circ g=$ $\operatorname{deg} f+\operatorname{deg} g$ whenever this makes sense) and all identity maps are of degree 0 .

(2) There is a $\mathbb{Z}$-action $(m, \mathcal{O}) \mapsto \mathcal{O}\{m\}$, called "grading shift by $m$ ", on the objects of $\mathcal{C}$. As plain Abelian groups, morphisms are unchanged by this action, $\operatorname{Mor}\left(\mathcal{O}_{1}\left\{m_{1}\right\}, \mathcal{O}_{2}\left\{m_{2}\right\}\right)=\operatorname{Mor}\left(\mathcal{O}_{1}, \mathcal{O}_{2}\right)$. But gradings do change under the action; so if $f \in \operatorname{Mor}\left(\mathcal{O}_{1}, \mathcal{O}_{2}\right)$ and $\operatorname{deg} f=d$, then as an element of $\operatorname{Mor}\left(\mathcal{O}_{1}\left\{m_{1}\right\}, \mathcal{O}_{2}\left\{m_{2}\right\}\right)$ the degree of $f$ is $d+m_{2}-m_{1}$. 
We note that if an pre-additive category only has the first property above, it can be 'upgraded' to a category $\mathcal{C}^{\prime}$ that has the second property as well. Simply let the objects of $\mathcal{C}^{\prime}$ be "artificial" $\mathcal{O}\{m\}$ for every $m \in \mathbb{Z}$ and every $\mathcal{O} \in \operatorname{Obj}(\mathcal{C})$ and it is clear how to define a $\mathbb{Z}$-action on $\operatorname{Obj}\left(\mathcal{C}^{\prime}\right)$ and how to define and grade the morphisms of $\mathcal{C}^{\prime}$. In what follows, we will suppress the prime from $\mathcal{C}^{\prime}$ and just call is $\mathcal{C}$; that is, whenever the morphism groups are graded, we will allow ourselves to grade-shift the objects of $\mathcal{C}$.

We also note that if $\mathcal{C}$ is a graded category then $\operatorname{Mat}(\mathcal{C})$ can also be considered as a graded category (a matrix is considered homogeneous of degree $d$ iff all its entries are of degree $d$ ). Complexes in $\operatorname{Kom}(\mathcal{C})($ or $\operatorname{Kom}(\operatorname{Mat}(\mathcal{C})))$ become graded in a similar way.

Definition 6.2 Let $C \in \operatorname{Mor}\left(\mathcal{C}_{o} b^{3}(B)\right)$ be a cobordism in a cylinder, with $|B|$ vertical boundary components on the side of the cylinder. Define $\operatorname{deg} C:=$ $\chi(C)-\frac{1}{2}|B|$, where $\chi(C)$ is the Euler characteristic of $C$.

Exercise 6.3 Verify that the degree of a cobordism is additive under vertical compositions (compositions of morphisms in $\mathcal{C}_{o b} b^{3}(B)$ ) and under horizontal compositions (using the planar algebra structure of Section 5 ), and verify that the degree of a saddle is $-1(\operatorname{deg})-(=-1)$ and that the degree of a cap/cup is $+1(\operatorname{deg}, \mathfrak{Q}=\operatorname{deg} Q=+1)$. As every cobordism is a vertical/horizontal composition of copies of $)-(, \alpha$ and $Q$, this allows for a quick computation of degrees.

Using the above definition and exercise we know that $\mathcal{C}_{o b}{ }^{3}$ is a graded category, and as the $S, T$ and $4 T u$ relations are degree-homogeneous, so is $\mathcal{C}_{o b} b_{/ l}^{3}$. Hence so are the target categories of [.], the categories $\operatorname{Kob}_{/ h}=\operatorname{Kom}_{/ h}\left(\operatorname{Mat}\left(\mathcal{C}_{o b}{ }^{3}\right)\right)$ and $\mathrm{Kob}_{/ h}=\mathrm{Kob} /($ homotopy $)$.

Definition 6.4 Let $T$ be a tangle diagram with $n_{+}$positive crossings and $n_{-}$negative crossings. Let $K h(T)$ be the complex whose chain spaces are $K h^{r}(T):=[T]\left\{r+n_{+}-n_{-}\right\}$and whose differentials are the same as those of $\llbracket T \rrbracket:$

$$
\begin{array}{rccccc}
{[T \rrbracket:} & {[T]^{-n^{-}}} & \longrightarrow & \cdots & \longrightarrow & {[T]^{n_{+}}} \\
K h(T): & {[T]^{-n^{-}}\left\{n_{+}-2 n_{-}\right\}} & \longrightarrow & \cdots & \longrightarrow & {[T]^{n_{+}}\left\{2 n_{+}-n_{-}\right\}}
\end{array}
$$




\section{Theorem 3}

(1) All differentials in $K h(T)$ are of degree 0.

(2) $K h(T)$ is an invariant of the tangle $T$ up to degree-0 homotopy equivalences. That is, if $T_{1}$ and $T_{2}$ are tangle diagrams which differ by some Reidemeister moves, then there is a homotopy equivalence $F: K h\left(T_{1}\right) \rightarrow$ $K h\left(T_{2}\right)$ with $\operatorname{deg} F=0$.

(3) Like [·], Kh descends to an oriented planar algebra morphism $(\mathcal{T}(s)) \rightarrow$ $(\operatorname{Kob}(s))$, and all the planar algebra operations are of degree 0 .

Proof The first assertion follows from $\operatorname{deg} x=-1$ and from the presence of $r$ in the degree shift $\left\{r+n_{+}-n_{-}\right\}$defining $K h$. The second assertion follows from a quick inspection of the homotopy equivalences in the proofs of invariance under $R 1$ and $R 2$ in section 4.3 and the third assertion follows from the corresponding one for [.] and from the additivity of $n_{+}$and $n_{-}$under the planar algebra operations.

\section{Applying a TQFT and obtaining a homology the- ory}

So $K h$ is an up-to-homotopy invariant of tangles, and it has excellent composition properties. But its target space, Kob, is quite unmanageable - given two formal complexes, how can one decide if they are homotopy equivalent?

In this section we will see how to take the homology of $K h(T)$. In this we lose some of the information in $K h(T)$ and lose its excellent composition properties. But we get a computable invariant, strong enough to be interesting.

Let $\mathcal{A}$ be some arbitrary Abelian category 8 . Any functor $\mathcal{F}: \mathcal{C}_{0} b_{/ l}^{3} \rightarrow \mathcal{A}$ extends right away (by taking formal direct sums into honest direct sums) to a functor $\mathcal{F}: \operatorname{Mat}\left(\mathcal{C}_{o b}^{3}{ }_{l}\right) \rightarrow \mathcal{A}$ and hence to a functor $\mathcal{F}: \operatorname{Kob} \rightarrow \operatorname{Kom}(\mathcal{A})$. Thus for any tangle diagram $T, \mathcal{F} K h(T)$ is an ordinary complex, and applying $\mathcal{F}$ to all homotopies, we see that $\mathcal{F} K h(T)$ is an up-to-homotopy invariant of the tangle $T$. Thus the isomorphism class of the homology $H(\mathcal{F} K h(T))$ is an invariant of $T$.

If in addition $\mathcal{A}$ is graded and the functor $\mathcal{F}$ is degree-respecting in the obvious sense, then the homology $H(\mathcal{F} K h(T))$ is a graded invariant of $T$. And if $\mathcal{F}$ is

\footnotetext{
${ }^{8}$ You are welcome to think of $\mathcal{A}$ as being the category of vector spaces or of $\mathbb{Z}_{-}^{-}$ modules.
} 
only partially defined, say on $\mathcal{C}_{o b}^{3}{ }_{l}(\emptyset)$, we get a partially defined homological invariant - in the case of $\mathcal{C}_{o b}^{3}{ }_{l l}(\emptyset)$, for example, its domain will be knots and links rather than arbitrary tangles.

We wish to postpone a fuller discussion of the possible choices for such a functor $\mathcal{F}$ to Section 9 and just give the standard example here. Our example for $\mathcal{F}$ will be a $T Q F T$ - a functor on $\mathcal{C}_{o b} b^{3}(\emptyset)$ valued in the category $\mathbb{Z}$ Mod of graded $\mathbb{Z}$-modules which maps disjoint unions of to tensor products. It is enough to define $\mathcal{F}$ on the generators of $\mathcal{C}_{o b} b^{3}(\emptyset)$ : the object $\bigcirc$ (a single circle) and the morphisms $, \propto, Q, C$ and $\curvearrowright$ (the cap, cup, pair of pants and upside down pair of pants).

Definition 7.1 Let $V$ be the graded $\mathbb{Z}$-module freely generated by two elements $\left\{v_{ \pm}\right\}$with $\operatorname{deg} v_{ \pm}= \pm 1$. Let $\mathcal{F}$ be the TQFT defined by $\mathcal{F}(\bigcirc)=V$ and by $\mathcal{F}(\not \subset)=\epsilon: \mathbb{Z} \rightarrow V, \mathcal{F}(\Theta)=\eta: V \rightarrow \mathbb{Z}, \mathcal{F}(\subsetneq)=\Delta: V \rightarrow V \otimes V$ and $\mathcal{F}(\circ)=m: V \otimes V \rightarrow V$, where these maps are defined by

$$
\begin{aligned}
& \mathcal{F}(\mathscr{O})=\epsilon:\left\{1 \mapsto v_{+}\right. \\
& \mathcal{F}(\bigcirc)=\eta:\left\{v_{+} \mapsto 0 \quad v_{-} \mapsto 1\right. \\
& \mathcal{F}(\circlearrowleft)=\Delta:\left\{\begin{array}{l}
v_{+} \mapsto v_{+} \otimes v_{-}+v_{-} \otimes v_{+} \\
v_{-} \mapsto v_{-} \otimes v_{-}
\end{array}\right. \\
& \mathcal{F}\left(\bigcirc_{-}\right)=m: \begin{cases}v_{+} \otimes v_{-} \mapsto v_{-} & v_{+} \otimes v_{+} \mapsto v_{+} \\
v_{-} \otimes v_{+} \mapsto v_{-} & v_{-} \otimes v_{-} \mapsto 0 .\end{cases}
\end{aligned}
$$

Proposition 7.2 $\mathcal{F}$ is well defined and degree-respecting. It descends to a functor $\mathcal{C o b}_{/ l}^{3}(\emptyset) \rightarrow \mathbb{Z M o d}$.

Proof It is well known that $\mathcal{F}$ is well defined - ie, that it respects the relations between our set of generators for $\mathcal{C} o b^{3}$, or the relations defining a Frobenius algebra. See eg, 10. It is easy to verify that $\mathcal{F}$ is degree-respecting, so it only remains to show that $\mathcal{F}$ satisfies the $S, T$ and $4 T u$ relations:

- S. A sphere is a cap followed by a cup, so we have to show that $\eta \circ \epsilon=0$. This holds.

- T. A torus is a cap followed by a pair of pants followed by an upside down pair of pants followed by a cup, so we have to compute $\eta \circ m \circ \Delta \circ \epsilon$. That's not too hard:

$$
1 \stackrel{\epsilon}{\longmapsto} v_{+} \stackrel{\Delta}{\longmapsto} v_{+} \otimes v_{-}+v_{-} \otimes v_{+} \stackrel{m}{\longmapsto} v_{-}+v_{-} \stackrel{\eta}{\longmapsto} 1+1=2 .
$$


- 4Tu. We will show that the equality $L=R$ holds in $V^{\otimes 4}$, where $L$ is given by $(\mathcal{F}(\curvearrowright \odot \odot)+\mathcal{F}(\bigcirc \bigcirc \bigcirc))(1)$ and likewise $R$ is given by

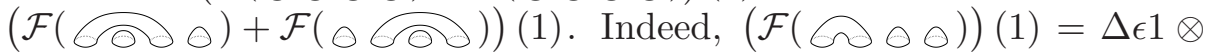
$\epsilon 1 \otimes \epsilon 1=v_{-} \otimes v_{+} \otimes v_{+} \otimes v_{+}+v_{+} \otimes v_{-} \otimes v_{+} \otimes v_{+}=: v_{-+++}+v_{+-++}$ and similarly $(\mathcal{F}(\bigcirc \bigcirc \bigcirc))(1)=v_{++-+}+v_{+++-}$and so $L=v_{-+++}+$ $v_{+-++}+v_{++-+}+v_{+++-}$. A similar computation shows $R$ to be the same.

Thus following the discussion at the beginning of this section, we know that for any $r$ the homology $H^{r}(\mathcal{F} K h(K))$ is an invariant of the knot or link $K$ with values in graded $\mathbb{Z}$-modules.

A quick comparison of the definitions shows that $H^{\star}(\mathcal{F} K h(K))$ is equal to Khovanov's categorification of the Jones polynomial and hence that its graded Euler characteristic is the Jones polynomial $\hat{J}$ (see [10, 3]). In my earlier paper 3. I computed $H^{\star}(\mathcal{F} K h(K)) \otimes \mathbb{Q}$ for all prime knots and links with up to 11 crossings and found that it is strictly a stronger knot and link invariant than the Jones polynomial. (See some further computations and conjectures at [12, 19]).

\section{Embedded cobordisms}

\subsection{Statement}

Let $\operatorname{Cob}^{4}(\emptyset)$ be the category whose objects are oriented based 9 knot or link diagrams in the plane, and whose morphisms are 2-dimensional cobordisms between such knot/link diagrams, generically embedded in $\mathbb{R}^{3} \times[0,1]$. Let $\mathcal{C}_{o b}{ }_{i i}^{4}(\emptyset)$ be the quotient of $\mathcal{C}_{o b} b^{4}(\emptyset)$ by isotopies (the $/ i$ stands for "modulo isotopies").

Note that the endomorphisms in $\operatorname{Cob}_{/ i}^{4}(\emptyset)$ of the empty link diagram are simply $2-$ knots, 2-dimensional knots (or links) in $\mathbb{R}^{3} \times(0,1) \equiv \mathbb{R}^{4}$. Hence much of what we will say below specializes to 2 -knots. Some wonderful drawings of $2-$ knots and

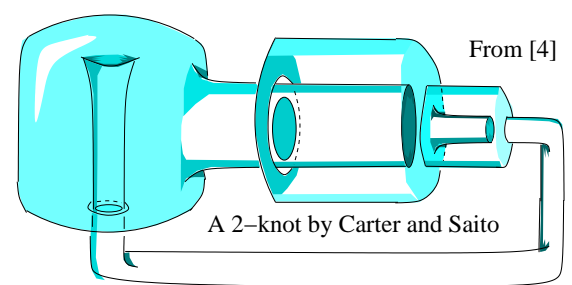
other cobordisms in 4-dimensional space are in the book by Carter and Saito, 4.

\footnotetext{
9 "Based" means that one of the crossings is starred. The only purpose of the basing is to break symmetries and hence to make the composition of morphisms unambiguous. For most purposes the basing can be ignored.
} 

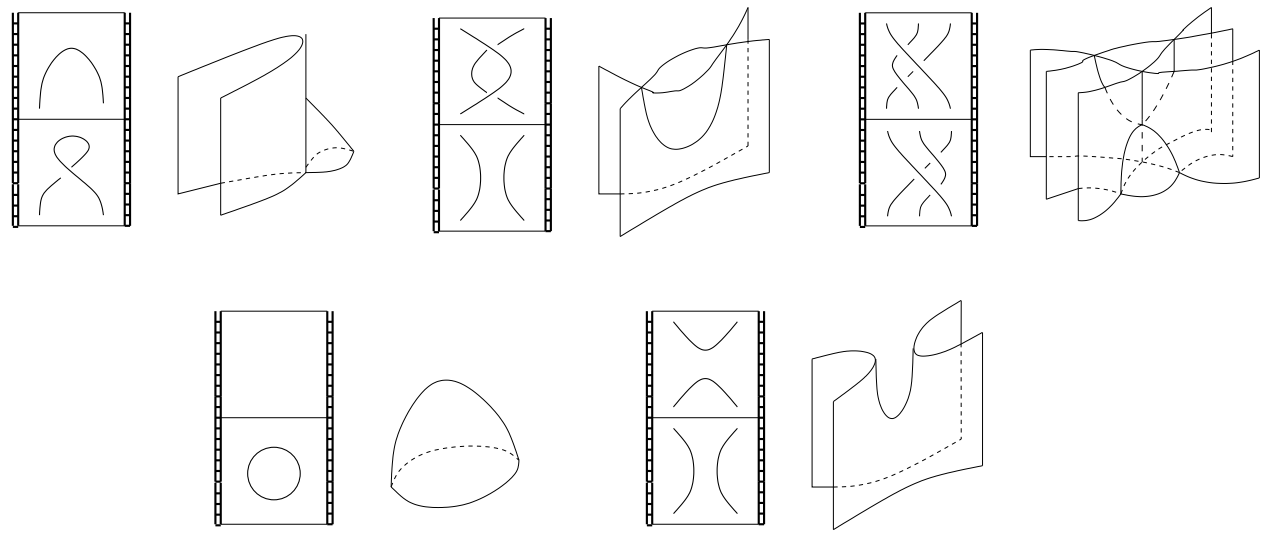

Figure 10: Elementary string interactions as movie clips and 3D projections of their $4 \mathrm{D}$ realizations, taken from 4 . (All clips are reversible.)

Thinking of the last coordinate in $\mathbb{R}^{3} \times[0,1]$ as time and projecting $\mathbb{R}^{3}$ down to the plane, we can think of every cobordism in $\mathcal{C} b^{4}(\emptyset)$ as a movie whose individual frames are knot/link diagrams (with at most finitely many singular exceptions). And if we shoot at a sufficiently high frame rate, then between any consecutive frames we will see (at most) one of the "elementary string interactions" of Figure 10 - a Reidemeister move, a cap or a cup, or a saddle. Thus the category $\mathcal{C}_{o b} b^{4}(\emptyset)$ is generated by the cobordisms corresponding to the three Reidemeister moves and by the cobordisms $, \alpha, Q$ and $)-($ now thought of as living in $4 D$ ).

We now define a functor $K h: \mathcal{C} b^{4}(\emptyset) \rightarrow \operatorname{Kob}(\emptyset)$. On objects, we've defined $K h$ already as the (formal) Khovanov homology of a given knot/link diagram. On the generating morphisms of $\mathcal{C}^{4} b^{4}(\emptyset)$ we define $K h$ as follows:

- Reidemeister moves go to the chain complex morphisms inducing the homotopy equivalences between the 'before' and 'after' complexes, as constructed within the proof of the invariance theorem (Theorem 1) in Section 4.3 .

- The cobordism $)(:)(\rightarrow \asymp$ induces a morphism $\llbracket-(\rrbracket: \llbracket)(\rrbracket \rightarrow \llbracket こ \rrbracket$ just as within the proof of invariance under R3, and just as there it can be interpreted in a 'skein theoretic' sense, where each symbol $)(),($ or $\asymp$ represents a small neighborhood within a larger context. But $[K \rrbracket$ differs from $K h(K)$ only by degree shifts, so the cobordism $)(:)(\rightarrow \asymp$ also induces a morphism $K h(-): K h()() \rightarrow K h(\asymp)$, as required. 
- Likewise, the cobordisms $\not: \emptyset \rightarrow \bigcirc$ and $Q: \bigcirc \rightarrow \emptyset$ induce morphisms of complexes $K h(\propto): K h(\emptyset) \rightarrow K h(\bigcirc)$ and $K h(\bigcirc): K h(\bigcirc) \rightarrow K h(\emptyset)$ (remember to interpret all this skein-theoretically — so the $\emptyset$ symbols here don't mean "the empty set", but just "the empty addition to some existing knot/link").

Theorem 4 Up to signs, Kh descends to a functor $K h: \mathcal{C o b}_{/ i}^{4}(\emptyset) \rightarrow \mathrm{Kob}_{/ h}(\emptyset)$. Precisely, let $\mathrm{Kob}_{/ \pm}$denote the projectivization of Kob — same objects, but every morphism is identified with its negative, and likewise let Kob $/ \pm h$ denote

the projectivization of $\mathrm{Kob}_{/ h}$. Then $K h$ descends to a functor $K h$ : $\mathcal{C o b}_{/ i}^{4}(\emptyset) \rightarrow$ $\mathrm{Kob}_{/ \pm h}(\emptyset)$.

The key to the proof of this theorem is to think locally. We need to show that circular movie clips in the kernel of the projection $\mathcal{C}_{o b}{ }^{4} \rightarrow \mathcal{C}_{o b} b_{i i}^{4}$ (such as the one in Equation (10) map to \pm 1 in $\operatorname{Kob}(\emptyset)$. As we shall see, the best way to do so is to view such a clip literally, as cobordisms between tangle diagrams, rather than symbolically, as skein-theoretic fragments of "bigger" cobordisms between knot/link diagrams.

Cobordisms between tangle diagrams compose in many ways to produce bigger cobordisms between tangle diagrams and ultimately to produce cobordisms between knot/link diagrams or possibly even to produce 2-knots. Cobordisms between tangle diagrams (presented, say, by movies) can be concatenated to give longer movies provided the last frame of one movie is equal to the first frame of the following movie. Thus cobordisms between tangle diagrams form a category. Cobordisms between tangle diagrams can also be composed like tangles, by placing them next to each other in the plane and connecting ends using planar arc diagrams. Hence cobordisms between tangle diagrams also form a planar algebra.

Thus our first task is to discuss those 'things' (called "canopolies" below) which are both planar algebras and categories. Ultimately we will prove that $K h$ is a morphism of canopolies between the canopoly of tangle cobordisms and an appropriate canopoly of formal complexes.

\subsection{Canopolies and a better statement}

Definition 8.1 Let $\mathcal{P}=(\mathcal{P}(k))$ be a planar algebra. A canopoly over $\mathcal{P}$ is a collection of categories $\mathcal{C}(k)$ indexed by the non-negative integers so that $\operatorname{Obj}(\mathcal{C}(k))=\mathcal{P}(k)$, so that the sets $\operatorname{Mor}(\mathcal{C}(k))$ of all morphisms between all 
objects of $\mathcal{C}(k)$ also form a planar algebra, and so that the planar algebra operations commute with the category operations (the compositions in the various categories). A morphism between a canopoly $\mathcal{C}^{1}$ over $\mathcal{P}^{1}$ and a canopoly $\mathcal{C}^{2}$ over $\mathcal{P}^{2}$ is a collection of functors $\mathcal{C}^{1}(k) \rightarrow \mathcal{C}^{2}(k)$ which also respect all the planar algebra operations. In a similar manner one may define 'oriented' canopolies $(\mathcal{C}(s))$ over oriented planar algebras $(\mathcal{P}(s))$ and morphisms between such canopolies. Every unoriented canopoly can also be regarded as an oriented one by setting $\mathcal{P}(s):=\mathcal{P}(|s|)$ and $\mathcal{C}(s):=\mathcal{C}(|s|)$ and otherwise ignoring all orientations.

A good way to visualize a canopoly is to think of $(\operatorname{Mor}(\mathcal{C}(k)))$ as a collection of 'cans' with labels in $(\mathcal{P}(k))$ on the tops and bottoms and with $k$ vertical lines on the sides, along with compositions rules that allow as to compose cans vertically when their tops/bottoms match and horizontally as in a planar algebra, and so that the vertical and horizontal compositions commute.

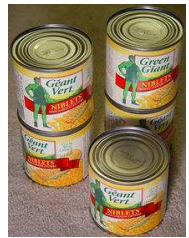

Example 8.2 $\mathcal{C} O b^{3}$ and $\mathcal{C} o b^{3}$ are canopolies over the planar algebra of crossingless tangles. A typical 'can' is shown on the right.

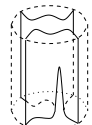

Example 8.3 For any finite set $B \subset S^{1}$ let $\mathcal{C}_{o b}{ }^{4}(B)$ be the category whose objects are tangle diagrams in the unit disk $D$ with boundary $B$ and whose morphisms are generic 2 -dimensional cobordisms between such tangle diagrams embedded in $D \times(-\epsilon, \epsilon) \times[0,1]$ with extra boundary (beyond the top and the bottom) the vertical

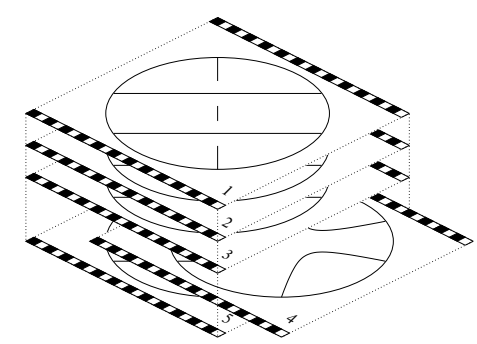
lines $B \times(-\epsilon, \epsilon) \times[0,1]$. For any non-negative $k$, let $\mathcal{C}_{o} b^{4}(k)$ be $\mathcal{C}_{0} b^{4}(B)$ with $B$ some $k$-element set in $S^{1}$. Then $\mathcal{C}_{0} b^{4}:=\bigcup_{k} \mathcal{C}_{o b} b^{4}(k)$ is a canopoly over the planar algebra of tangle diagrams. With generic cobordisms visualized as movies, a can in $\mathcal{C}_{o} b^{4}$ becomes a vertical stack of frames, each one depicting an intermediate tangle diagram. In addition, we $\bmod \mathcal{C}_{o b} b^{4}$ out by isotopies and call the resulting canopoly $\mathcal{C}_{o b}{ }_{/ i}^{4}$.

Example 8.4 The collection $\mathrm{Kob}=\bigcup_{k} \operatorname{Kob}(k)$, previously regarded only as a planar algebra, can also be viewed as a canopoly. In this canopoly the 'tops' and 'bottoms' of cans are formal complexes and the cans themselves are morphisms between complexes. Likewise $\mathrm{Kob}_{/ h}=\mathrm{Kob} /$ (homotopy), Kob $/ \pm=\mathrm{Kob} / \pm 1$ and $\mathrm{Kob}_{/ \pm h}:=\mathrm{Kob}_{/ h} / \pm 1$ can be regarded as canopolies. 
We note that precisely the same constructions as in Section 8.1 though replacing the empty boundary $\emptyset$ by a general $k$ element boundary $B$, define a functor $K h_{0}: \mathcal{C}_{o b} b^{4}(B) \rightarrow \operatorname{Kob}(B)$ for any $B$. As these constructions are local, it is clear that these functors assemble together to form a canopoly morphism $K h_{0}: \mathcal{C}_{o b} b^{4} \rightarrow$ Kob from the canopoly of movie presentations of four dimensional cobordisms between tangle diagrams to the canopoly of formal complexes and morphisms between them.

We also note that the notion of a graded canopoly can be defined along the lines of Section [6 - grade the cans (but not the planar algebras of the "tops" and "bottoms") and insist that all the can composition operations be degreeadditive. One easily verifies that all the above mentioned canopolies are in fact graded, with the gradings induced from the gradings of $\mathcal{C} o b^{3}$ and of $\mathcal{C}_{o b}{ }^{4}\left(\mathcal{C}_{o b} b^{3}\right.$ was given a grading in Definition 6.2 and Exercise 6.3. and the same definition and exercise can be applied without changing a word to $\mathcal{C}_{o b}{ }^{4}$ ). Clearly $K h_{0}$ is degree preserving.

The following theorem obviously generalizes Theorem 4 and is easier to prove:

Theorem $5 \quad K h_{0}$ descends to a degree preserving canopoly morphism $K h$ : $\mathrm{Cob}_{/ i}^{4} \rightarrow \mathrm{Kob}_{/ \pm h}$ from the canopoly of four dimensional cobordisms between tangle diagrams to the canopoly of formal complexes with up to sign and up to homotopy morphisms between them.

\subsection{Proof}

We just need to show that $K h_{0}$ respects the relations in the kernel of the projection $\mathcal{C} b^{4} \rightarrow \mathcal{C} o b^{4}{ }_{i}$. These are the "movie moves" of Carter and Saito [4, reproduced here in Figures 11, 12, and 13, In principle, this is a routine verification. All that one needs to do is to write down explicitly the morphism of complexes corresponding to each of the clips in those figures, and to verify that these morphisms are homotopic to identity morphisms (in some cases) or to each other (in other cases).

But this isn't as simple as it sounds, as many of the complexes involved are quite complicated. The worst is of course $\mathrm{MM}_{10}$ of Figure 12 - each frame in that clip involves a 6-crossing tangle, and hence a 6-dimensional cube of 64 smoothings, and each of the 8 moves in $\mathrm{MM}_{10}$ is an $R 3$ move, so the morphism corresponding to it originates from the morphism displayed in Figure 9 Even if in principle routine, it obviously isn't a simple task to show that the composition 

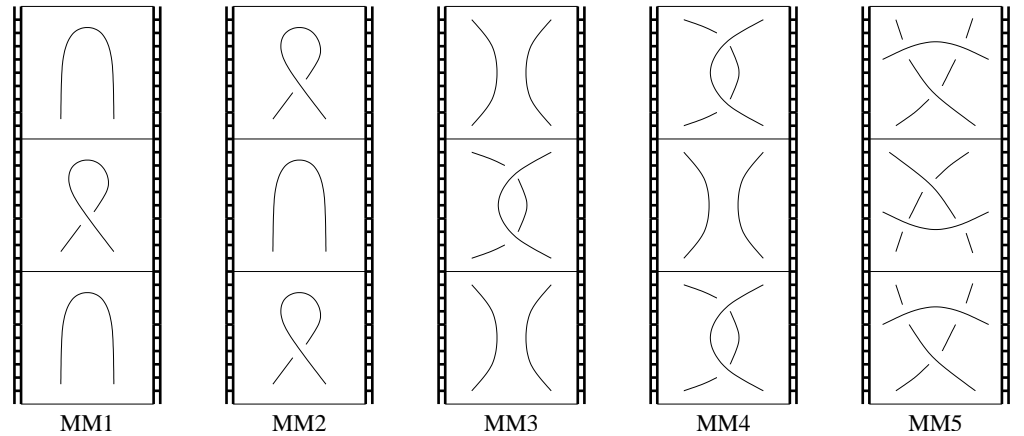

Figure 11: Movie moves as in Carter and Saito 4. Type I: Reidemeister and inverses. These short clips are equivalent to "do nothing" identity clips.
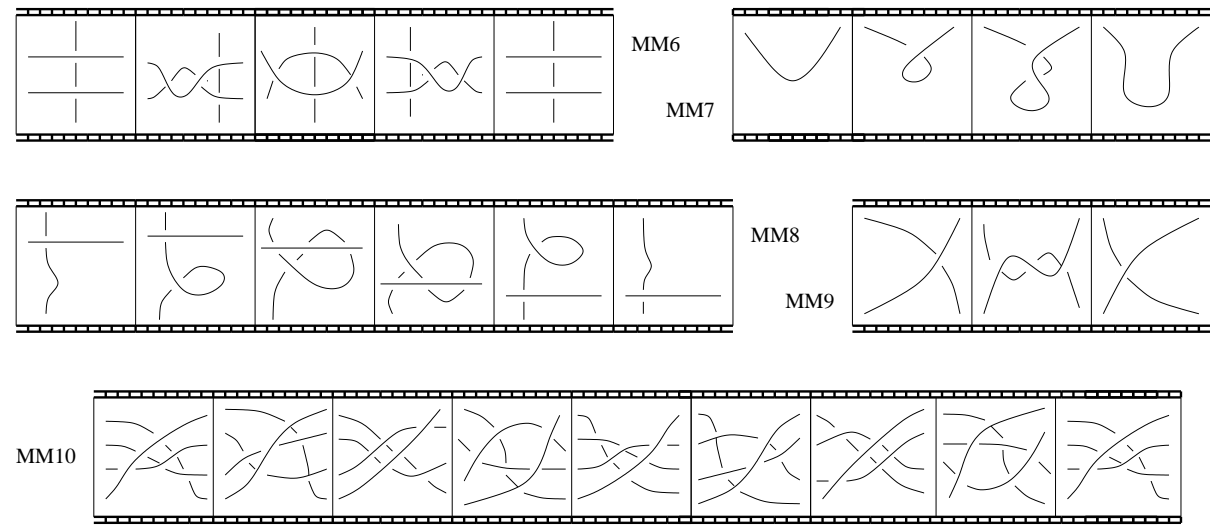

Figure 12: Movie moves as in Carter and Saito 4]. Type II: Reversible circular clips - equivalent to identity clips.
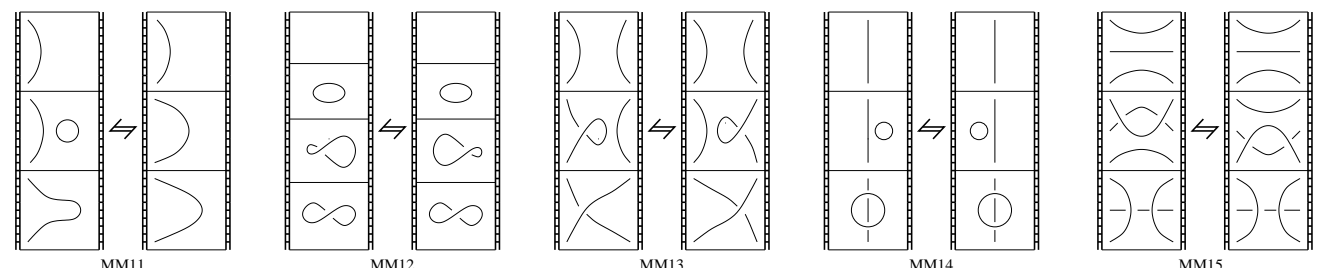

Figure 13: Movie moves as in Carter and Saito 4]. Type III: Non-reversible clips (can be read both from the top down and from the bottom up). 
of 8 such beasts is homotopic to the identity automorphism (of a 6-dimensional cube).

This is essentially the approach taken by Jacobsson in [6], where he was able to use clever tricks and clever notation to reduce this complexity significantly, though much complexity remains. At the end of the day the theorem is proven by carrying out a number of long computations, but it remains a mystery whether these computations had to work out, or is it just a concurrence of lucky coincidences.

Our proof of Theorem 5 is completely different, though it is very similar in spirit to Khovanov's proof [13. The key to our proof is the fact that the complexes corresponding to many of the tangles appearing in Figures 11, 12, and 13 simply have no automorphisms other than up-to-homotopy \pm 1 multiples of the identity, and hence $K h_{0}$ has no choice but to send the clips in Figures [1] and 12 to up-to-homotopy \pm 1 multiples of the identity.

We start with a formal definition of "no automorphisms" and then prove 4 short lemmas that together show that there are indeed many tangles whose corresponding complexes have "no automorphisms":

Definition 8.5 We say that a tangle diagram $T$ is $K h$-simple if every degree 0 automorphism of $K h(T)$ is homotopic to a \pm 1 multiple of the identity. (An automorphism, in this context, is a homotopy equivalence of $K h(T)$ with itself).

Lemma 8.6 Pairings are $K h$-simple (a pairing is a tangle that has no crossings and no closed components, so it is just a planar pairing of its boundary points).

Proof If $T$ is a pairing then $K h(T)$ is the 0 -dimensional cube of the $2^{0}$ smoothings of $T$ - namely, it is merely the one step complex consisting of $T$ alone at height 0 and of no differentials at all. A degree 0 automorphism of this complex is a formal $\mathbb{Z}$-linear combination of degree 0 cobordisms with top and bottom equal to $T$.

Let us take one such cobordism and call it $C$. By the definition of degrees in Section [6 it follows that $C$ must have Euler characteristic equal to the number of its boundary components (which is the same as the number of components of $T$ and half the number of boundary points of $T$ ). If $C$ has no connected components with no boundary, this forces $C$ to be a union of

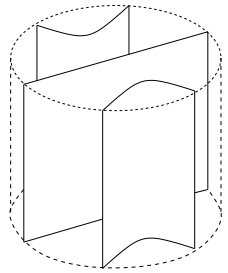
disks embedded vertically (as "curtains") as on the right. Any tori (whose Euler 
characteristic is 0$)$ in $C$ can be reduced using the $T$ relation and any higher genus boundaryless components (with negative Euler characteristic) must be balanced against spherical components (whose Euler characteristic is positive). But the latter are 0 by the $S$ relation.

Hence $C$ is the identity and so $K h(T)$ is a multiple of the identity. But being invertible it must therefore be a \pm 1 multiple of the identity.

Lemma 8.7 If a tangle diagram $T$ is $K h$-simple and a tangle diagram $T^{\prime}$ represents an isotopic tangle, then $T^{\prime}$ is also $K h$-simple.

Proof By the invariance of $K h$ (Theorems 1 and 2), the complexes $K h\left(T^{\prime}\right)$ and $K h(T)$ are homotopy equivalent. Choose a homotopy equivalence $F: K h\left(T^{\prime}\right) \rightarrow$ $K h(T)$ between the two (with up-to-homotopy inverse $\left.G: K h(T) \rightarrow K h\left(T^{\prime}\right)\right)$, and assume $\alpha^{\prime}$ is a degree 0 au-

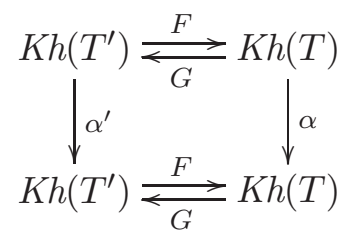
tomorphism of $K h\left(T^{\prime}\right)$. As $T$ is $K h$-simple, we know that $\alpha:=F \alpha^{\prime} G$ is homotopic to $\pm I$ and so $\alpha^{\prime} \sim G F \alpha^{\prime} G F=G \alpha F \sim \pm G F \sim$ $\pm I$, and so $T^{\prime}$ is also $K h$-simple.

Now let $T$ be a tangle and let $T X$ be a tangle obtained from $T$ by adding one extra crossing $X$ somewhere along the boundary of $T$, so that exactly two (adjacent) legs of $X$ are connected to $T$ and two remain free. This operation of adjoining a crossing is "invertible"; one can adjoin the inverse crossing $X^{-1}$ to get $T X X^{-1}$ which is isotopic to the original tangle $T$. This fact is utilized in the following two lemmas to show that $T$ is $K h$-simple iff $T X$ is $K h$-simple.

But first a word about notation. In a canopoly there are many operations and two of them will be used in the following proof.

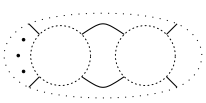

Within that proof we will denote the horizontal composition of putting things side by side by simple juxtaposition (more precisely, this is the planar algebra operation corresponding to the planar arc diagram on the right). Thus starting with a tangle $T$ and a crossing $X$ we get $T X$ as in the previous paragraph. The vertical composition of putting things one atop the other will be denoted by $\circ$.

Lemma 8.8 If $T X$ is $K h$-simple then so is $T$. 
Proof Let $\alpha: K h(T) \rightarrow K h(T)$ be a degree 0 automorphism. Using the planar algebra operations we adjoin a crossing $X$ on the right to $T$ and to $\alpha$ to get an automorphism $\alpha I_{X}: K h(T X) \rightarrow K h(T X)$ (here $I_{X}$ denotes the identity automorphism of $K h(X))$. As $T X$ is $K h$-simple, $\alpha I_{X} \sim \pm I$. We now adjoin the inverse $X^{-1}$ of $X$ and find that $\alpha I_{X X^{-1}}: K h\left(T X X^{-1}\right) \rightarrow K h\left(T X X^{-1}\right) \sim$ $\pm I$. But $X X^{-1}$ is differs from $\asymp$ by merely a Reidemeister move, so let $F: K h(\asymp) \rightarrow K h\left(X X^{-1}\right)$ be the homotopy equivalence between $K h(\asymp)$ and $K h\left(X X^{-1}\right)$ (with up-to-homotopy inverse $\left.G: K h\left(X X^{-1}\right) \rightarrow K h(\asymp)\right)$. Now $\alpha=\alpha I_{K h(\asymp)} \sim \alpha(G \circ F)=\left(I_{K h(T)} G\right) \circ\left(\alpha I_{X X^{-1}}\right) \circ\left(I_{K h(T)} F\right) \sim \pm\left(I_{K h(T)} G\right) \circ I \circ$ $\left(I_{K h(T)} F\right)= \pm I_{K h(T)}(G \circ F) \sim \pm I$, and so $T$ is $K h$-simple.

Lemma 8.9 If $T$ is $K h$-simple then so is $T X$.

Proof Assume $T$ is $K h$-simple. By Lemma 8.7 so is $T X X^{-1}$. Using Lemma 8.8 we can drop one crossing, the $X^{-1}$, and find that $T X$ is $K h$-simple.

We can finally get back to the proof of Theorem 5 . Recall that we have to show that $K h_{0}$ respects each of the movie moves $\mathrm{MM}_{1}$ through $\mathrm{MM}_{15}$ displayed in Figures [11, 12 and 13. These movie moves can be divided into three types as follows.

Type I Performing a Reidemeister and then its inverse (Figure 11) is the same as doing nothing. Applying $K h_{0}$ we clearly get morphisms that are homotopic to the identity - this is precisely the content of Theorem 3

Type II The reversible circular movie moves ("circular clips") of Figure 12 are equivalent to the "do nothing" clips that have the same initial and final scenes. This is the hardest and easiest type of

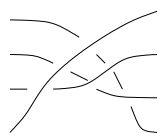
movie moves - the hardest because it includes the vicious $\mathrm{MM}_{10}$. The easiest because given our machinery the proof reduces to just a few sentences. Indeed, $K h_{0}\left(\mathrm{MM}_{10}\right)$ is an automorphism of $K h(T)$ where $T$ is the tangle beginning and ending the clip $\mathrm{MM}_{10}$. But using Lemma 8.9 we can peel off the crossings of $T$ one by one until we are left with a crossingless tangle (in fact, a pairing), which by Lemma 8.6 is $K h$-simple. So $T$ is also $K h$-simple and hence $K h_{0}\left(\mathrm{MM}_{10}\right) \sim$ $\pm I$ as required. The same argument works for $\mathrm{MM}_{6}$ through $\mathrm{MM}_{9}$. (And in fact, the same argument also works for $\mathrm{MM}_{1}$ through $\mathrm{MM}_{5}$, though as seen above, these movie moves afford an even easier argument).

Type III The pairs of equivalent clips appearing in Figure 13. With some additional effort one can adapt the proof for the type II movie moves to work here as well, but given the low number of crossings involved, the brute force approach becomes sufficiently gentle here. Indeed, we argue as follows. 
- For $\mathrm{MM}_{11}$ : Going down along the left side of $\mathrm{MM}_{11}$ we get a morphism $F: K h(\Omega) \rightarrow K h(\Omega)$. Both $K h(0)$ and $K h(\Omega)$ are one-step complexes, $\_$and $\_$respectively, and $F$ is just the cobordism $-0 \circ \sqrt{*}=8$

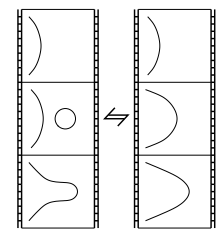
which is isotopic to the identity cobordism $\rightarrow>$. Going up along $\mathrm{MM}_{11}$, you just have to turn all these figures upside down.

- For $\mathrm{MM}_{12}$ : At the top of $\mathrm{MM}_{12}$ we see the empty tangle $\emptyset$ and $K h(\emptyset)=(\emptyset)$ is the one-step complex whose only "chain group" is the empty smoothing $\emptyset$. At the bottom, $K h(\infty)$ is the two step complex $\bigcirc \bigcirc \rightarrow \bigcirc$. Going down the right side of $\mathrm{MM}_{12}$ starting at $K h(\emptyset)$ we land in the height 0

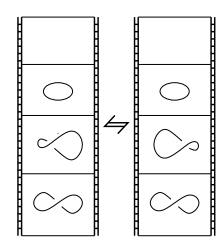
part $\bigcirc \bigcirc$ of $K h(\infty)$ and as can be seen from the proof of invariance under $R 1$, the resulting morphism $\emptyset \rightarrow \bigcirc \bigcirc$ is the difference $F_{R}=\oslash \otimes-\infty$. Likewise going along the left side of $\mathrm{MM}_{12}$ we get the difference $F_{L}=\otimes \ominus-\infty$. We leave it as an exercise to the reader to verify that modulo the $4 \mathrm{Tu}$ relation $F_{L}+F_{R}=0$ (hint: Equation (4) below) and hence the two side of $\mathrm{MM}_{12}$ are the same up to a sign. Going up $\mathrm{MM}_{12}$ is even easier.

- For $\mathrm{MM}_{13}$ : The height 0 part of $K h(\times)$ is )( so going down the two sides of $\mathrm{MM}_{13}$ we get two morphisms $)(\rightarrow)($, and both are obtained from the morphism $F^{0}$ of Figure 5 by composing it with an extra saddle. Tracing it through, we find that the left morphism is $\left.F_{L}=\right)$ e $-H$ and the

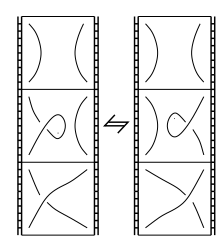
right morphism is $\left.F_{R}=\right) \theta(-)$ (here all cobordisms are shown from above and denotes a vertical curtain with an extra handle attached and $\mathcal{H}$ denotes two vertical curtain connected by a horizontal tube). We leave it as an exercise to the reader to verify that modulo the $4 T u$ relation $F_{L}+F_{R}=0$ (hint: (4)) and hence the two side of $\mathrm{MM}_{13}$ are the same up to a sign. Going up $\mathrm{MM}_{13}$ is even easier. 
- For $\mathrm{MM}_{14}$ : The two height 0 parts of $K h\left(\frac{1}{1}\right)$ are $\mid \circ$ and 이, and using the map $F$ of Figure 6 we see that the four morphisms $|\rightarrow| 0$ and $\mid \rightarrow$ o| obtained by tracing $\mathrm{MM}_{14}$ from the top to the bottom either on the left or on the right are all simple "circle creation" cobordisms (ie, caps) along with a vertical curtain. In particular, the left side and the

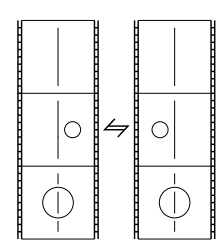
right side of $\mathrm{MM}_{14}$ produce the same answer. A similar argument works for the way up $\mathrm{MM}_{14}$.

- For $\mathrm{MM}_{15}$ : Quite nicely, going down the two sides of $\mathrm{MM}_{15}$ we get the two morphisms $\Psi_{L}$ and $\Psi_{R}$ of Figure 7 and these two are the same. Going up $\mathrm{MM}_{15}$ we get, in a similar manner, the two sides of the other variant of the $R 3$ move, as at the end of the invariance under $R 3$ proof on just above Lemma 4.5 .

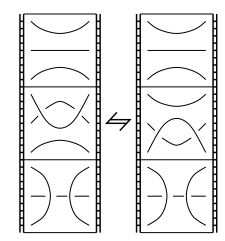

This concludes the proof of Theorems 5 and 4.

\section{$9 \quad$ More on $\mathcal{C o b}_{/ l}^{3}$}

In Section 7 we've seen how a functor $\mathcal{C} b^{3}{ }_{l} \rightarrow \mathbb{Z}$ Mod can take our theory (which now includes Theorems 4 and 5 as well) into something more computable. Thus we seek to construct many such functors. We start, right below, with a systematic construction of such "tautological" functors. Then in Sections 9.1 9.3 we will discuss several instances of the tautological construction, leading back to the original Khovanov theory (Section 9.1), to the Lee [14 variant of the original Khovanov theory (Section 9.2) and to a new variant defined only over the two element field $\mathbb{F}_{2}$ (Section 9.3).

Definition 9.1 Let $B$ be a set of points in $S^{1}$ and let $\mathcal{O}$ be an object of $\mathcal{C}_{o b}^{3}{ }_{l l}(B)$ (ie, a smoothing with boundary $B$; often if $B=\emptyset$ we will choose $\mathcal{O}$ to be the empty smoothing). The tautological functor $\mathcal{F}_{\mathcal{O}}: \mathcal{C o b}_{l}^{3} \rightarrow \mathbb{Z M o d}$ is defined on objects by $\mathcal{F}_{\mathcal{O}}\left(\mathcal{O}^{\prime}\right):=\operatorname{Mor}\left(\mathcal{O}, \mathcal{O}^{\prime}\right)$ and on morphisms by composition on the left. That is, if $F: \mathcal{O}^{\prime} \rightarrow \mathcal{O}^{\prime \prime}$ is a morphism in $\operatorname{Cob}_{/ l}^{3}(B)$ then $\mathcal{F}_{\mathcal{O}}(F): \operatorname{Mor}\left(\mathcal{O}, \mathcal{O}^{\prime}\right) \rightarrow \operatorname{Mor}\left(\mathcal{O}, \mathcal{O}^{\prime \prime}\right)$ maps $G \in \operatorname{Mor}\left(\mathcal{O}, \mathcal{O}^{\prime}\right)$ to $F \circ G \in$ $\operatorname{Mor}\left(\mathcal{O}, \mathcal{O}^{\prime \prime}\right)$.

At the moment we don't know the answer to the following question. 
Problem 9.2 Is the tautological construction faithful? Is there more information in $K h$ beyond its composition with tautological functors? Beyond the homology of its composition with tautological functors?

The groups of morphisms of $\mathcal{C} o b^{3}$ appear to be difficult to study. Hence we will often simplify matters a bit by composing tautological functors with some extra functors that forget some information. Examples follow below.

\subsection{Cutting necks and the original Khovanov homology theory}

As a first example we take $B=\emptyset$ and $\mathcal{O}=\emptyset$, we forget all 2-torsion by tensoring with some ground ring in which $2^{-1}$ exists $\left(\right.$ eg, $\left.\mathbb{Z}_{(2)}:=\mathbb{Z}[1 / 2]\right)$ and we mod out by all surfaces with genus greater than 1 :

$$
\mathcal{F}_{1}\left(\mathcal{O}^{\prime}\right):=\mathbb{Z}_{(2)} \otimes_{\mathbb{Z}} \operatorname{Mor}\left(\emptyset, \mathcal{O}^{\prime}\right) /((g>1)=0) .
$$

Taking $C$ to be a disjoint union of two twice-punctured disks in the specification of the $4 T u$ relation in Section 4.1.2 we get the neck cutting relation

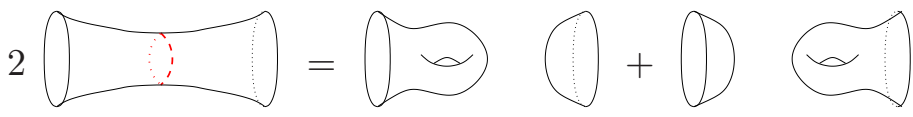

If 2 is invertible, the neck cutting relation means that we can "cut open" any tube inside a cobordism (replacing it by handles that are localized to one side of the tube). Repeatedly cutting tubes in this manner we see that $\mathbb{Z}_{(2)} \otimes_{\mathbb{Z}}$ $\operatorname{Mor}\left(\emptyset, \mathcal{O}^{\prime}\right)$ is generated by cobordisms in which every connected component touchs at most one boundary curve. Further reducing using the $S, T$ and $((g>$ $1)=0)$ relations we get to cobordisms in which every connected component has precisely one boundary curve and is either of genus 0 or of genus 1 . So if $\mathcal{O}^{\prime}$ is made of $k$ curves then $\mathcal{F}_{1}\left(\mathcal{O}^{\prime}\right)=V^{\otimes k}$ where $V$ is the $\mathbb{Z}_{(2)}$-module generated by $v_{+}:=\varnothing$ and by $v_{-}:=\frac{1}{2} \otimes$.

Exercise 9.3 Verify that with this basis for $V$ the (reduced) tautological functor $\mathcal{F}_{1}$ becomes the same as the functor $\mathcal{F}$ of Definition [7.1, and hence once again we reproduce the original Khovanov homology theory. 
Hint 9.4 Using the braket notation of quantum mechanics, the bras dual to the kets $v_{+}=|\Theta\rangle$ and $v_{-}=\left|\frac{1}{2} \otimes\right\rangle$ are $\left\langle\frac{1}{2} \otimes\right|$

\begin{tabular}{c|c|c} 
glue & $|\Theta\rangle$ & $\left|\frac{1}{2} \Theta\right\rangle$ \\
\hline$\left\langle\frac{1}{2} \Theta\right|$ & $\frac{1}{2}\langle\Theta\rangle=1$ & $\frac{1}{4}\langle\otimes\rangle=0$
\end{tabular}
and $\langle\Theta|$ respectively. Thus, for example, the coefficient of $v_{-}$within the product $v_{-} v_{+}$is $\left\langle\oslash|\ominus \theta| \frac{1}{2} \odot \Theta\right\rangle=\frac{1}{2}\langle$ cactus $\rangle=\frac{1}{2}\langle\Theta\rangle=1$.

\subsection{Genus 3 and Lee's theory}

At first glance it may appear that the relation $((g>1)=0)$ was unnecessary in the above discussion - every high genus surface contains several "necks" and we can cut those using (4) to get lower genus surfaces. This clearly works if the genus $g$ is high enough to start with ( $g \geq 4$ is enough). Cutting the obvious neck in the genus 2 surface $\rightleftharpoons$ and reducing tori using the $T$ relation we find that $\Theta=0$ automatically. But the genus 3 surface with no boundary $\odot$ cannot be reduced any further.

Thus setting

$$
\mathcal{F}_{2}\left(\mathcal{O}^{\prime}\right):=\mathbb{Z}_{(2)} \otimes_{\mathbb{Z}} \operatorname{Mor}\left(\emptyset, \mathcal{O}^{\prime}\right) /(\bigodot \infty=8)
$$

we find that as a $\mathbb{Z}_{(2)}$-module $\mathcal{F}_{2}\left(\mathcal{O}^{\prime}\right)$ is as in the previous example and as in Definition 7.1 (except that the grading is lost), but $\Delta$ and $m$ are modified as follows:

$$
\Delta_{2}:\left\{\begin{array}{l}
v_{+} \mapsto v_{+} \otimes v_{-}+v_{-} \otimes v_{+} \\
v_{-} \mapsto v_{-} \otimes v_{-}+v_{+} \otimes v_{+}
\end{array} \quad m_{2}: \begin{cases}v_{+} \otimes v_{-} \mapsto v_{-} & v_{+} \otimes v_{+} \mapsto v_{+} \\
v_{-} \otimes v_{+} \mapsto v_{-} & v_{-} \otimes v_{-} \mapsto v_{+}\end{cases}\right.
$$

This is precisely the Lee [14 variant of the original Khovanov theory (used by Rasmussen [16] to give a purely combinatorial proof of the Milnor conjecture).

\subsection{Characteristic 2 and a new homology theory}

The other extreme is to tensor multiply with $\mathbb{F}_{2}$ (ie, to take all linear combinations with coefficients in $\mathbb{F}_{2}$ ). In this case it is convenient to take the "target object" $\mathcal{O}$ to be a single boundariless cycle $\bigcirc$ and set

$$
\mathcal{F}_{3}\left(\mathcal{O}^{\prime}\right):=\mathbb{F}_{2} \otimes_{\mathbb{Z}} \operatorname{Mor}\left(\bigcirc, \mathcal{O}^{\prime}\right) .
$$



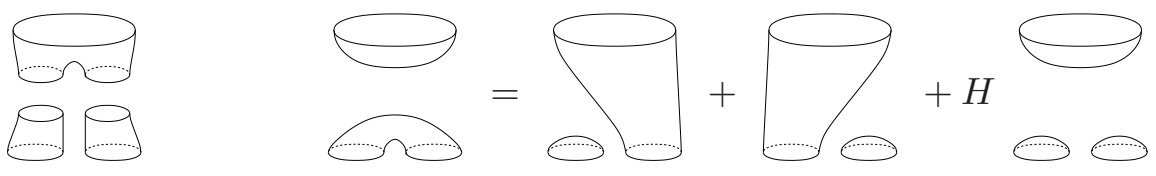

Figure 14: A local picture and the corresponding $4 T u$ relation (over $\mathbb{F}_{2}[H]$, so signs can be disregarded and handles replaced by $H$ 's).

Over $\mathbb{F}_{2}$ the neck drops off the neck cutting relation (4) and what remains is a relation (shown on the right) saying that

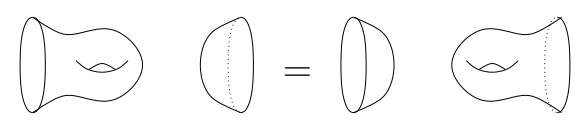
handles can be moved from one component to another. We introduce a new variable $H$ with $\operatorname{deg} H=-2$ and with the relation $H \Theta=\Theta$, so $H$ stands for "a handle inserted somewhere (anywhere) into a cobordism". As modules over $\mathbb{F}_{2}[H]$ the morphisms of $\mathbb{F}_{2} \otimes \mathcal{C} o b_{/ l}^{3}$ are generated by cobordisms with no handles, ie, by punctured spheres. (An exception needs to be made for cobordisms with no boundary at all, in $\mathcal{C}_{0} b_{l l}^{3}(\emptyset)$. Our morphisms all have a source $\mathcal{O}=\bigcirc$ and hence they always have at least one boundary component so this exception is irrelevant in what follows.)

Let $\bigcirc^{k}=\bigcirc \bigcirc \cdots \bigcirc(k$ circles $)$ be a typical object of $\mathcal{C}_{o b}^{3}{ }_{l l}(\emptyset)$ and consider $\mathcal{F}_{3}\left(\bigcirc^{k}\right)=\mathbb{F}_{2} \otimes_{\mathbb{Z}} \operatorname{Mor}\left(\bigcirc, \bigcirc^{k}\right)$. Over $\mathbb{F}_{2}[H]$ this module is generated by surfaces whose components are punctured spheres with an overall number of $k+1$ punctures, $k$ of which corresponding to

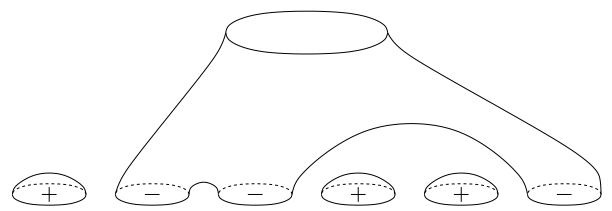

Figure 15: $v_{+--++-} \in \mathcal{F}_{3}\left(\bigcirc^{6}\right)$ the $k$ circles in the "target object" $\bigcirc^{k}$ and a special puncture corresponding to the "source object" $\bigcirc$. The $4 \mathrm{Tu}$ relation of Figure 14 can be used to dissolve any component that has more than one puncture and that does not contain the special puncture into components that either contain just one puncture or contain the special puncture. Hence $\mathcal{F}_{3}\left(\bigcirc^{k}\right)$ is generated by cobordisms such as the one in Figure 15, in which there is a special spherical component whose boundary contains the source object (and possibly several other circles from the target object) and possibly several disjoint disks that cap the remaining parts of the target object. As in Figure 15, these generators are in a natural bijective correspondence with the elements of $V^{\otimes k}$ where $V$ (as before) is generated by two elements $v_{ \pm}$with $\operatorname{deg} v_{ \pm}= \pm 1$ (though this time $v_{+}$and $v_{-}$cannot be identified with the disk and half a punctured torus as in Sections 9.1 and 9.2). 
Exercise 9.5 Check that with this identification of $\mathcal{F}_{3}\left(\bigcirc^{k}\right)$ with $V^{\otimes k}$, the generating morphisms of $\operatorname{Cob}_{/ l}^{3}(\emptyset)$ map to

$$
\begin{aligned}
& \Delta_{3}:\left\{\begin{array}{l}
v_{+} \mapsto v_{+} \otimes v_{-}+v_{-} \otimes v_{+}+H v_{+} \otimes v_{+} \\
v_{-} \mapsto v_{-} \otimes v_{-}
\end{array}\right. \\
& m_{3}: \begin{cases}v_{+} \otimes v_{-} \mapsto v_{-} & v_{+} \otimes v_{+} \mapsto v_{+} \\
v_{-} \otimes v_{+} \mapsto v_{-} & v_{-} \otimes v_{-} \mapsto H v_{-} .\end{cases}
\end{aligned}
$$

We know nothing in general about the homology of $\mathcal{F}_{3} K h(L)$ associated with a knot/link $L$ and/or its relationship with the original Khovanov homology $H(\mathcal{F} K h(L))$. Let us describe here the results of some sporadic computations that we have performed. We took $H=1$ in the above formulas (losing the grading, of course) and obtained a filtered theory, where $\mathcal{G}_{\geq j} \mathcal{F}_{3}^{1} K h(L)$ denotes the subcomplex of $\mathcal{F}_{3}^{1} K h(L)$ made of chains of degrees greater than or equal to $j$. We then computed the Betti numbers $b_{r j}^{3}(L):=\operatorname{dim}_{\mathbb{F}_{2}} H^{r}\left(\mathcal{G}_{\geq j} \mathcal{F}_{3}^{1} K h(L)\right)$ and compared them with the Betti numbers of the original Khovanov homology over $\mathbb{Q}$ and over $\mathbb{F}_{2}$ (ie, with $b_{r j}^{\mathbb{Q}}(L):=\operatorname{dim}_{\mathbb{Q}} H(\mathbb{Q} \otimes \mathcal{F} K h(L))$ and with $b_{r j}^{\mathbb{F}_{2}}(L):=$ $\operatorname{dim}_{\mathbb{F}_{2}} H\left(\mathbb{F}_{2} \otimes \mathcal{F} K h(L)\right)$ ), for several knots and links with up to 10 crossings. The results of these computations are best displayed as two dimensional arrays of numbers, as in Figure 16.

Readers familiar with 12, 19, 3, will notice that every "knight move" in the array describing $b_{r j}^{\mathbb{Q}}$ gets replaced by a "tetris piece" in the array for $b_{r j}^{\mathbb{F}_{2}}$ and by a "shifted pawn move" in the array for $b_{r j}^{3}$, while

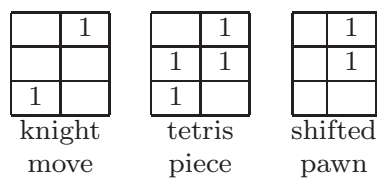

the special pawn in column 0 of $b_{r j}^{\mathbb{Q}}$ stays put for $b_{r j}^{\mathbb{F}_{2}}$ and gets replaced by a column with entries $(1,2,2, \ldots)$ in the array for $b_{r j}^{3}$. This observation repeats for all the knots that we have tested but we don't know a general explanation (though see Conjecture 1 below).

\section{Trace groups, Euler characteristics and skein mod- ules}

We wish to push the relationship between $K h$ and the Jones polynomial a little further 10 . Both $K h$ and $\hat{J}$ can be computed locally, first for small tangles

\footnotetext{
10 This section was inspired by a talk AS Sikora gave at the George Washington University on May 2004 and by [2]. The results in [2] partially overlap ours.
} 


\begin{tabular}{|c|c|c|c|c|c|}
\hline$j$ & -2 & -1 & 0 & 1 & 2 \\
\hline 5 & & & & & $1,1,1$ \\
\hline 3 & & & & $0,1,0$ & $0,1,1$ \\
\hline 1 & & & $1,1,1$ & $1,1,0$ & \\
\hline-1 & & $1,1,1$ & $1,1,2$ & \multicolumn{3}{|c|}{} \\
\hline-3 & $0,1,0$ & $0,1,1$ & $0,0,2$ & \multirow{2}{*}{} \\
\hline-5 & $1,1,0$ & & $0,0,2$ & \\
\hline$<-5$ & & & $0,0,2$ & \\
\hline
\end{tabular}

\begin{tabular}{|c|c|c|c|c|c|c|c|c|}
\hline$j$ & -4 & -3 & -2 & -1 & 0 & 1 & 2 & 3 \\
\hline 9 & & & & & & & & $1,1,1$ \\
\hline 7 & & & & & & & $1,2,1$ & $0,1,1$ \\
\hline 5 & & & & & & $1,2,1$ & $1,2,1$ & \\
\hline 3 & & & & & $2,3,2$ & $1,2,1$ & & \\
\hline 1 & & & & $1,3,1$ & $2,4,3$ & & & \\
\hline-1 & & & $1,2,1$ & $2,3,1$ & $1,1,2$ & & & \\
\hline-3 & & $1,2,1$ & $1,2,1$ & & $0,0,2$ & & & \\
\hline-5 & $0,1,0$ & $1,2,1$ & & & $0,0,2$ & & & \\
\hline-7 & $1,1,0$ & & & & $0,0,2$ & & & \\
\hline
\end{tabular}

Figure 16: The knots $4_{1}$ and $10_{136}$ (as drawn by 18) and their Betti numbers in a tabular form. Each box contains the three numbers $b_{r j}^{\mathbb{Q}}, b_{r j}^{\mathbb{F}_{2}}$ and $b_{r j}^{3}$ in order. Empty boxes mean that all three Betti numbers are zero.

and then, by composing small tangles, the computations can be carried out for bigger tangles and whole links. But the relationship with the Jones polynomial discussed in Section 7 only works at the level of whole links. In this section we will see how an appropriate notion of "Euler characteristic" intertwines $K h$ and $\hat{J}$ while respecting the planar algebra structure (so progressive computations of $K h$ and $\hat{J}$ "run in tandem").

\subsection{Traces}

We first have to generalize the notions of Euler characteristic, dimension and trace to complexes over arbitrary pre-additive categories.

Definition 10.1 A "trace" on a category $\mathcal{C}$ with values in an Abelian group $A$ is an additive map $\tau: \bigoplus_{\mathcal{O} \in \operatorname{Obj}(\mathcal{C})} \operatorname{Mor}(\mathcal{O}, \mathcal{O}) \rightarrow A$ defined on all the endomorphisms of objects in $\mathcal{C}$ and with values in $A$, which satisfies the "trace relation" $\tau(F G)=\tau(G F)$ whenever $F$ and $G$ are morphisms so that both $F G$ and $G F$ are endomorphisms (ie, whenever $F: \mathcal{O}_{1} \rightarrow \mathcal{O}_{2}$ and $G: \mathcal{O}_{2} \rightarrow \mathcal{O}_{1}$ for some $\left.\mathcal{O}_{1,2} \in \operatorname{Obj}(\mathcal{C})\right)$. 
This definition is analogous to the standard definition of the trace of a matrix, which is defined only for square matrices but satisfies the trace relation $\operatorname{tr}(F G)=\operatorname{tr}(G F)$ even for non-square $F$ and $G$, provided both $F G$ and $G F$ are square.

Exercise 10.2 If $\tau$ is a trace on $\mathcal{C}$ and if $\left(F_{i j}\right)$ is an endomorphism of some object in $\operatorname{Mat}(\mathcal{C})$, set $\tau\left(\left(F_{i j}\right)\right):=\sum_{i} \tau\left(F_{i i}\right)$ and show that the newly defined $\tau$ is a trace on $\operatorname{Mat}(\mathcal{C})$.

Assuming the presence of some fixed trace $\tau$ on a pre-additive category $\mathcal{C}$, we can now proceed to define dimensions, Euler characteristics and Lefschetz numbers:

Definition 10.3 The dimension (more precisely, the " $\tau$-dimension") of an object $\mathcal{O}$ of $\mathcal{C}$ or in $\operatorname{Mat}(\mathcal{C})$ is the trace of the identity: $\operatorname{dim}_{\tau} \mathcal{O}:=\tau\left(I_{\mathcal{O}}\right)$. If $\left(\Omega^{r}\right)$ is a complex in $\operatorname{Kom}(\mathcal{C})$ or in $\operatorname{Kom}(\operatorname{Mat}(\mathcal{C}))$ we define its Euler characteristic (more precisely, its " $\tau$-Euler characteristic") to be

$$
\chi_{\tau}\left(\left(\Omega^{r}\right)\right):=\sum_{r}(-1)^{r} \operatorname{dim}_{\tau} \Omega^{r} .
$$

Finally, if $F=\left(F^{r}\right)$ is an endomorphism of $\Omega$ we define its Lefschetz number (or " $\tau$-Lefschetz number") to be $\tau(F):=\sum_{r}(-1)^{r} \tau\left(F^{r}\right)$. (All these quantities are members of the Abelian group $A$ which may or may not be our underlying group of scalars). Clearly, $\chi_{\tau}(\Omega)=\tau\left(I_{\Omega}\right)$.

We claim that Lefschetz number and Euler characteristics are homotopy invariant:

\section{Proposition 10.4}

(1) If $\Omega$ is a complex and $F$ and $G$ are homotopic endomorphisms $F, G: \Omega \rightarrow$ $\Omega$ then $\tau(F)=\tau(G)$.

(2) If the complexes $\Omega_{a}$ and $\Omega_{b}$ are homotopy equivalent then $\chi_{\tau}\left(\Omega_{a}\right)=$ $\chi_{\tau}\left(\Omega_{b}\right)$.

\section{Proof}

(1) Let $h$ be so that $F-G=h d+d h$. Then, using the trace relation for the last equality,

$$
\begin{gathered}
\tau(F)-\tau(G)=\sum_{r}(-1)^{r} \tau\left(F^{r}-G^{r}\right)=\sum_{r}(-1)^{r} \tau\left(h^{r+1} d^{r}+d^{r-1} h^{r}\right) \\
=\sum_{r}(-1)^{r} \tau\left(h^{r+1} d^{r}-d^{r} h^{r+1}\right)=0 .
\end{gathered}
$$


(2) If $F: \Omega_{a} \rightarrow \Omega_{b}$ and $G: \Omega_{b} \rightarrow \Omega_{a}$ induce the homotopy equivalence then $G F \sim I_{\Omega_{a}}$ and $F G \sim I_{\Omega_{b}}$ and so

$$
\chi_{\tau}\left(\Omega_{a}\right)=\tau\left(I_{\Omega_{a}}\right)=\tau(G F)=\tau(F G)=\tau\left(I_{\Omega_{b}}\right)=\chi_{\tau}\left(\Omega_{b}\right)
$$

(using the trace relation once more, for the middle equality)

\subsection{The trace group and the universal trace}

Given a pre-additive category $\mathcal{C}$ it is interesting to find all traces defined on it. Quite clearly, they all factor through the "universal trace" defined below:

Definition 10.5 The "trace group" $\Xi(\mathcal{C})$ is 11

$$
\Xi(\mathcal{C}):=\bigoplus_{\mathcal{O} \in \operatorname{Obj}(\mathcal{C})} \operatorname{Mor}(\mathcal{O}, \mathcal{O}) / \begin{aligned}
& \text { the trace relation: } F G=G F \text { whenever } \\
& F: \mathcal{O}_{1} \rightarrow \mathcal{O}_{2} \text { and } G: \mathcal{O}_{2} \rightarrow \mathcal{O}_{1}
\end{aligned}
$$

The map which takes any endomorphism in $\mathcal{C}$ to itself as a member of $\Xi(\mathcal{C})$ is denoted $\tau_{\star}$ and called "the universal trace of $\mathcal{C}$ ". (It is, of course, a trace). We denote dimensions and Euler characteristics defined using $\tau_{\star}$ (hence valued in $\Xi(\mathcal{C}))$ by $\operatorname{dim}_{\star}$ and $\chi_{\star}$.

Example 10.6 (Told by D Thurston; see also [20]) Let Mat $(\bullet)$ be the category whose objects are non-negative integers and whose morphisms are rectangular integer matrices of appropriate dimensions: $\operatorname{Mor}(n, m)=\{m \times n$ matrices $\}$. Then the trace group $\Xi(\operatorname{Mat}(\bullet))$ is generated by square matrices. The trace relation

$$
\left(\begin{array}{ll}
0 & f \\
0 & 0
\end{array}\right)=\left(\begin{array}{ll}
0 & f \\
0 & 0
\end{array}\right)\left(\begin{array}{ll}
0 & 0 \\
0 & 1
\end{array}\right)=\left(\begin{array}{ll}
0 & 0 \\
0 & 1
\end{array}\right)\left(\begin{array}{ll}
0 & f \\
0 & 0
\end{array}\right)=\left(\begin{array}{ll}
0 & 0 \\
0 & 0
\end{array}\right)
$$

allows us to cancel off-diagonal entries (perhaps after extending the relation by adding zero rows and columns). Diagonal matrices can be written as sums of diagonal matrices that have just one non-zero entry on the diagonal, and then empty row/column pairs can be removed using the trace relation

$$
\left(\begin{array}{ll}
g & 0 \\
0 & 0
\end{array}\right)=\left(\begin{array}{l}
g \\
0
\end{array}\right)\left(\begin{array}{ll}
1 & 0
\end{array}\right)=\left(\begin{array}{ll}
1 & 0
\end{array}\right)\left(\begin{array}{l}
g \\
0
\end{array}\right)=(g) .
$$

Thus $\Xi(\operatorname{Mat}(\bullet))$ is generated by the $1 \times 1$ matrix $(1)$, and so the ordinary matrix trace is up to scalars the unique trace on $\operatorname{Mat}(\bullet)$ and $\Xi(\operatorname{Mat}(\bullet))$ is isomorphic to $\mathbb{Z}$.

\footnotetext{
${ }^{11} \mathrm{~S}$ D Schack informed me that this notion is due to F W Lawvere and S H Schanuel. It is also the same as " 0 th Hochschild-Mitchell homology of a category with coefficients in itself", but it is not the same as the "Grothendieck group" of an Abelian category.
} 
Example 10.7 It is likewise easy to show that the trace group of the category of finite dimensional vector spaces and linear maps over some ground field $\mathbb{F}$ is $\mathbb{F}$ itself (generated by the identity on a one-dimensional vector space) and that (up to scalars) the unique trace on finite dimensional vector spaces is the ordinary trace.

Exercise 10.8 Refine the argument in Example 10.6 to show that $\Xi(\operatorname{Mat}(\mathcal{C}))$ $=\Xi(\mathcal{C})$ for any pre-additive category $\mathcal{C}$.

Example 10.9 Let Vect $_{0}$ be the category of finite dimensional graded vector spaces (over a field $\mathbb{F}$ ) with degree 0 morphisms between them. Then, loosely speaking, the different homogeneous components don't interact and so $\Xi$ (Vect $\left._{0}\right)$ has one generator in each degree. The generator in degree $m$ can be taken to be the identity on a one-dimensional graded vector space whose only non-zero homogeneous component is in degree $m$. Denoting this generator by $q^{m}$ we can identify $\Xi\left(\right.$ Vect $\left._{0}\right)$ with the ring of Laurent polynomials $\mathbb{F}\left[q, q^{-1}\right]$ (where $q$ is a formal variable which is best thought of as carrying degree 1). Thus in this case $\operatorname{dim}_{\star}$ and $\chi_{\star}$ are the $q$-dimension and $q$-Euler characteristic of the theory of finite dimensional graded vector spaces (denoted $q$ dim and $\chi_{q}$ in [3] ).

\subsection{The trace groups of $\mathcal{C} O b_{0 / l}^{3}$ and skein modules}

Theorems 10 2] and 3 all provide us with invariants of tangles with values in homotopy classes of complexes. In each case we can find the trace group of the category underlying the target complexes and then compute universal Euler characteristics, thus getting potentially simpler "non-homological" invariants. Here we only do it for the most refined of the three invariants, $K h$ of Theorem [3. though to keep things simpler, we also make the two simplifications of Section 9.1 - we forget all 2-torsion by tensoring with $\mathbb{Z}_{(2)}$ and we mod out by all surfaces with genus greater than 1 . As we shall see, this recovers the Jones polynomial for tangles.

Some preliminary definitions are required. Let $B_{k}$ be a collection of $k$ points in $S^{1}$. Recall from Section [6 that by allowing artificial degree shifts $\mathcal{C}_{o b}^{3}{ }_{/ l}\left(B_{k}\right)$ can be considered as a graded category and from Theorem 3 that in as much as $K h$ is concerned, we can restrict our attention to morphisms in $\mathcal{C}_{o b}{ }^{3}\left(B_{k}\right)$ that, degree shifts considered, are of degree 0 . Let $\mathcal{C} o b_{0 / l}^{3}$ be the restricted category, tensored with $\mathbb{Z}_{(2)}$ and modulo $((g>1)=0)$ : the objects of $\mathcal{C} o b_{0 / l}^{3}$ are animals of the form $S\{m\}$ where $S$ is a smoothing and $m$ is an integer indicating a 
formal degree shift, and the morphisms from $S_{1}\left\{m_{1}\right\}$ to $S_{2}\left\{m_{2}\right\}$ are $\mathbb{Z}_{(2)}$ linear combinations of cobordisms whose top is $S_{1}$ and bottom is $S_{2}$ and whose degree in the sense of Definition 6.2 is $m_{1}-m_{2}$, taken modulo the $S, T$, $4 T u$ and $((g>1)=0)$ relations. Let $\Xi_{k}$ be the trace group $\Xi\left(\mathcal{C o b}_{0 / l}^{3}(k)\right)$ of the category $\mathcal{C}_{0} b_{0 / l}^{3}$. The collection $\left(\Xi_{k}\right)$ inherits a planar algebra structure from the planar algebra structure of the morphisms of $\mathcal{C} o b^{3}$ and as the universal Euler characteristic is homotopy invariant, the first part of the following theorem is evident:

\section{Theorem 6}

(1) $\chi_{\star} \circ K h$ is an invariant of tangles with values in $\left(\Xi_{k}\right)$; in fact, $\chi_{\star} \circ K h$ is an oriented planar algebra morphism $(\mathcal{T}(s)) \rightarrow(\Xi(s)$ ) (where $\Xi(s):=$ $\Xi(|s|))$.

(2) $\chi_{\star} \circ \mathrm{Kh}$ is the Jones polynomial for tangles (proof follows the proof Proposition (10.10).

Recall from [15] that the Jones polynomial $\hat{J}$ for $k$-ended tangle 12 can be defined via the "skein relations" $\hat{J}$ : $\aleph \mapsto q)\left(-q^{2} \asymp\right.$ and $\left.\hat{J}: \boldsymbol{\lambda} \mapsto-q^{-2} \asymp+q^{-1}\right)($. It takes values in the "skein module" $\mathcal{S}_{k}$ for $k$-ended tangles - the collection of $\mathbb{Z}\left[q, q^{-1}\right]$-linear combinations of $k$-ended crossingless tangles modulo the extra relation $\bigcirc=q+q^{-1}$.

Proposition 10.10 The trace group $\Xi_{k}$ is naturally isomorphic to the skein module $\mathcal{S}_{k}$. If $S$ a $k$-ended crossingless tangle (ie, a smoothing), the isomorphism $\sigma: \mathcal{S}_{k} \rightarrow \Xi_{k}$ maps $q^{m} S$ to the identity automorphism of the object $S\{m\}$ of $\mathcal{C o b}_{0 / l}^{3}(k)$, regarded as a member of the trace group $\Xi_{k}=\Xi\left(\operatorname{Cob}_{0 / l}^{3}(k)\right)$.

Proof $\Xi_{k}$ is spanned by pairs $(S\{m\}, C)$ where $S$ is a $k$-ended smoothing, $m$ is an integer indicating a formal degree shift, and $C$ is a degree 0 cobordism $C: S \rightarrow S$. Such pairs are taken modulo the trace relation $F G=G F$ of Definition 10.5. We can visualize such a pair as a cylinder with $C$ inside, with $S$ on the top and at the bottom and with extra $q^{m}$ coefficient placed in front, (below left).
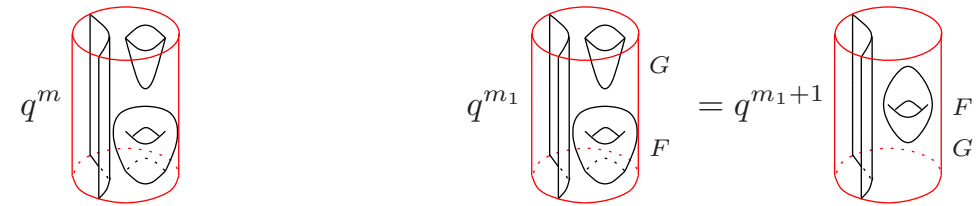

\footnotetext{
${ }^{12}$ Our slightly non-standard normalization was chosen to make the statement of Theorem [6] as simple as possible. For links, $\hat{J}(L)(q)=\left(q+q^{-1}\right) J(L)\left(q^{2}\right)$, where $J$ is the standard Jones polynomial, as normalized (say) in [9].
} 
Let $F: S_{1}\left\{m_{1}\right\} \rightarrow S_{2}\left\{m_{2}\right\}$ and $G: S_{2}\left\{m_{2}\right\} \rightarrow S_{1}\left\{m_{1}\right\}$ be arbitrary degree 0 morphisms in $\mathcal{C}_{0} b_{0 / l}^{3}(k)$. That is, $F$ and $G$ are degree $m_{1}-m_{2}$ and $m_{2}-m_{1}$ cobordisms within a cylinder (degrees measured as in Definition 6.2), with tops $S_{1}$ and $S_{2}$ and bottoms $S_{2}$ and $S_{1}$ (all respectively), see above right. The trace relation states that $C:=G F$, an endomorphism of $S_{1}\left\{m_{1}\right\}$, is equal to $F G$, which is an endomorphism of $S_{2}\left\{m_{2}\right\}$. Setting $m=m_{2}-m_{1}$ and visualizing as before, the trace relation becomes "cut a degree $m$ piece $G$ off the top of $C$ and reglue it at the bottom while multiplying by extra factor of $q^{m}$ ". With this interpretation of the trace relation it is easy to check that $\sigma$ respects the relation $\bigcirc=q+q^{-1}$ and is hence well defined. Indeed, using the neck cutting relation 4 , then the trace relation and then the $T$ relation we get:

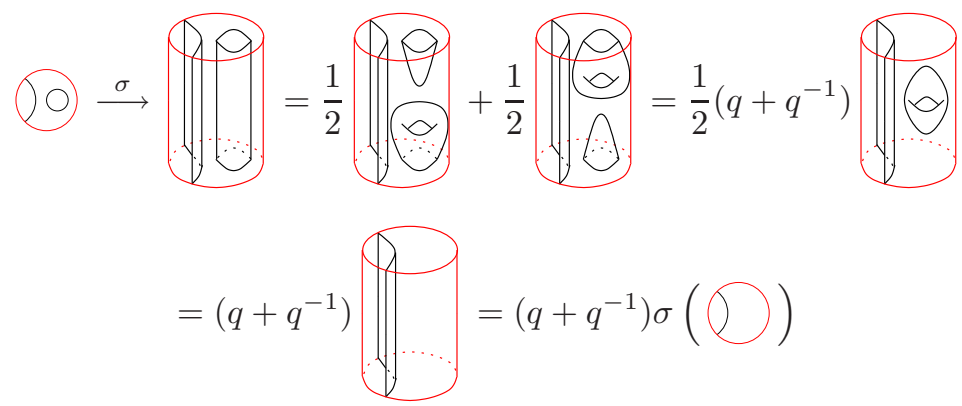

Now let $(S\{m\}, C)$ be a general element in $\Xi_{k}$. Cutting necks in $C$ using the neck cutting relation (4) as in Section 9.1 and the relations $S, T$ and $((g>1)=$ $0)$, we can assume without loss of generality that every connected component of $C$ has precisely one boundary component (which is a cycle on the boundary of the cylinder). Furthermore, using the trace relation as above, components attached to top boundary components can be brought to the bottom (perhaps at the cost of some powers of $q$ ), where they 'cancel' the corresponding bottom boundary components and create boundary-free components of $C$. And again, these can be removed using the $S, T$ and $((g>1)=0)$ relations. So without loss of generality $C$ just has $k / 2$ components with "rectangular" boundary, made of an arc on the top of the cylinder, an arc on the bottom, and two arcs on the sides. As $\operatorname{deg} C=0$ it follows from Definition 6.2 that all those components of $C$ must be disks, so $C$ is simply $S \times I$ for a cycle-free $k$-ended $S$. Hence $\sigma$ is surjective.

We leave it to the reader to verify that the procedure described in the previous paragraph does not depend on the choices within it (the only apparent choice is the ordering of the necks for cutting) and hence it defines a well-defined inverse for $\sigma$, concluding the proof of Proposition 10.10.

Proof of part (2) of Theorem [6] Given part (1) of the theorem, it is only 
necessary to verify part (2) in the case of crossingless tangles (which is tautological) the single-crossing tangles $\lambda$ and $\lambda$. The latter two cases follow immediately from definition of the Jones polynomial, $\chi \rightarrow q)\left(-q^{2} \asymp\right.$ and $\lambda \rightarrow$ $\left.-q^{-2} \asymp+q^{-1}\right)$ (, the corresponding evaluations of $K h$, $\rightarrow(\underline{)(\{1\}} \longrightarrow \asymp\{2\})$ and $\lambda \rightarrow(\asymp\{-2\} \longrightarrow \underline{(\{-1\}})$, the definition of the Euler characteristic $\chi_{\star}$ and the identification of $\mathcal{S}_{k}$ with $\Xi_{k}$ via $\sigma$.

\section{Odds and ends}

\subsection{A structural conjecture}

We say that a complex in $\operatorname{Kob}(\emptyset)$ is "basic" if up to degree and height shifts it is either one of the following two complexes:

- The one term complex $\Omega_{1}: 0 \longrightarrow 0 \longrightarrow 0$ whose only non-zero term is a smoothing consisting of a single circle.

- The two term complex $\Omega_{2}: 0 \longrightarrow 0 \stackrel{d}{\longrightarrow} \bigcirc \longrightarrow 0$ whose two nonzero terms are both smoothings consisting of a single circle and whose only non-zero differential $d=\varnothing$ is the genus 1 surface with a circle boundary at the top and a circle boundary at the bottom.

We say that a link $L$ with $c$ components is Khovanov-basic if the complex $K h(L)$ is homotopy equivalent to a direct sum of basic complexes and the number of $\Omega_{1}$ terms appearing is $2^{c-1}$.

One can verify that Khovanov-basic links have Betti numbers consistent with the knight-tetris-pawn observations of the previous section and with Lee's Theorem 5.1 (see [14). But the Betti numbers computed by Shumakovitch in 19, Appendices A.4 and A.5] show that some links are not Khovanov-basic.

Conjecture 1 Alternating links are Khovanov-basic (and so are many other links, but we'd rather remain uncommitted).

\subsection{Dotted cobordisms}

As an aside, in this section we briefly describe a weaker variant of our theory which on links is equivalent to the original Khovanov theory 13 but is still

\footnotetext{
${ }^{13}$ And so it does not project to Lee's theory or to the "new" theory of Sections 9.2 and 9.3
} 
rich enough for our tangles and cobordisms discussion to go through mostly unchanged.

Extend the category $\mathcal{C}_{o} b^{3}$ to a new category $\mathcal{C}_{0} b^{3}$ that has the same objects as $\mathcal{C} o b^{3}$ and nearly the same morphisms - the only difference is that we now allow "dots" (of degree -2) that can be marked on cobordisms and moved freely within each connected component of a given cobordisms. We then form the quotient category $\mathcal{C}_{0} b_{\bullet / l}^{3}$ by reducing $\mathcal{C}_{0} b_{\bullet}^{3}$ modulo the local relations

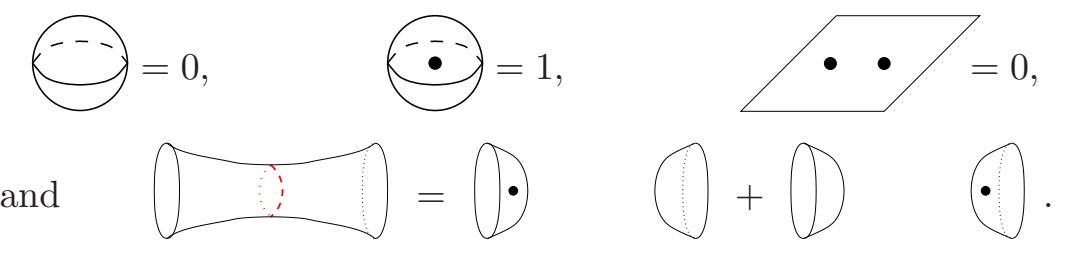

Pretty much everything done with $\mathcal{C}_{o} b_{/ l}^{3}$ can be repeated verbatim for $\mathcal{C}_{0} b_{\bullet / l}^{3}$ (indeed, the $S, T$ and $4 T u$ relations follow from the above relations), and in particular, the appropriately modified Theorems 1 through 6 hold. But now we have a stronger neck cutting relation and tubes and handles can be removed regardless of the ground ring. At the end we only need to consider cobordisms in which every connected component is either a disk or a singly-dotted disk, and these can be identified with $v_{+}$and $v_{-}$of Section 7 Applying a tautological functor as in Section 9 (with $\mathcal{O}=\emptyset$ ) we get back to the standard Khovanov homology without any restriction on the ground ring.

\subsection{Abstract cobordisms}

As defined, the cobordisms in the various variants of $\mathcal{C} o b^{3}$ considered in this paper are all embedded into cylinders, and hence a full classification of the morphisms in $\mathcal{C}^{3} b^{3}$ could be as complicated as knot theory itself. Two comments are in order.

- We could have just as well worked with un-embedded cobordisms cobordisms whose boundaries are embedded in cylinders so as to have gluing operations as required in this paper, but whose insides are "abstract surfaces". All the results of this paper continue to hold in this setting as well.

- In principle there are more embedded cobordisms than abstract cobordisms, so keeping track of the embeddings potentially yields a richer theory. Though if 2 is invertible then the neck cutting relation (4) shows that embedding information can be forgotten anyway and so in this case the two theories are the same. 


\subsection{Equivalent forms of the $4 T u$ relation}

We have chosen to present the $4 T u$ relation the way we did because in its present form it is handy to use within the proof Theorem 1 But there are several equivalent or nearly equivalent alternatives.

Proposition 11.1 If the number 2 is invertible, the neck cutting relation of Equation (4) is equivalent to the $4 \mathrm{Tu}$ relation.

Proof We've already seen in Equation 4 that the $4 T u$ relation implies the neck cutting relation regardless of the ground ring. To go the other way, use $2^{-1}$ and the neck cutting relation to cut the four tubes in the $4 \mathrm{Tu}$ relation and replace them with 8 handles. These eight handles cancel out in pairs.

Proposition 11.2 The following two three-site relations are equivalent to each other and to the $4 \mathrm{Tu}$ relation (over any ground ring):

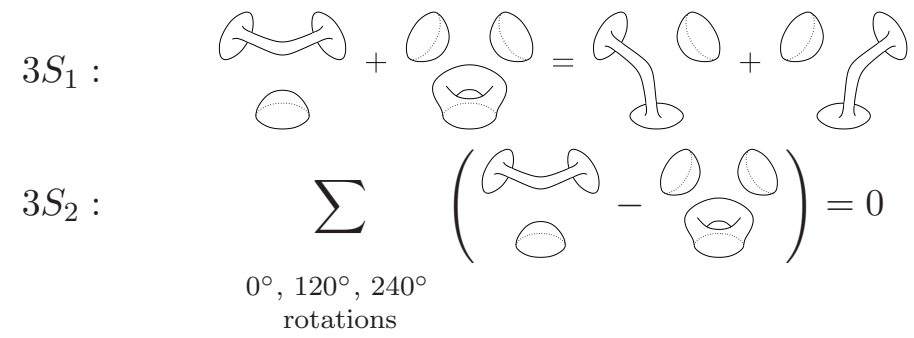

Proof $4 T u \Rightarrow 3 S_{1}$ : The relation $3 S_{1}$ is in fact the relation used in the proof of invariance under $R 1$; just as over there, it follows from the $4 \mathrm{Tu}$ relation by specializing the general $4 \mathrm{Tu}$ relation to the case when two of the disks $D_{1}$ through $D_{4}$ that appear in the definition of the $4 \mathrm{Tu}$ relation are on the same connected component of the "ambient" cobordism.

$3 S_{1} \Rightarrow 3 S_{2}$ : First, by putting the two upper disks in the definition of $3 S_{1}$ on the same connected component, we see that $3 S_{1}$ implies the neck cutting relation. Now subtract the neck cutting relation corresponding to the left-most tube in $3 S_{1}$ from the entire $3 S_{1}$ relation. The result is $3 S_{2}$.

$3 S_{2} \Rightarrow 4 T u$ : As in the previous case, by putting the two upper disks in the definition of $3 S_{2}$ on the same connected component, we see that $3 S_{2}$ implies the neck cutting relation. Now consider a cobordism $C$ with four special disks $D_{1}$ through $D_{4}$ as in the definition of the $4 \mathrm{Tu}$ relation. The $3 S_{2}$ relation applied to $C$ at sites $1,2,3$ is $C_{12}+C_{23}+C_{13}-H_{1}-H_{2}-H_{3}$ where $C_{i j}$ are as in 
the definition of the $4 \mathrm{Tu}$ relation and where $H_{i}$ is $C$ with a handle added at $D_{i}$. Likewise, the $3 S_{2}$ relation applied to $C$ at sites $2,3,4$ is $C_{23}+C_{34}+C_{24}-$ $H_{2}-H_{3}-H_{4}$. The sum of these two $3 S_{2}$ relations is $\left(C_{12}+C_{34}-C_{13}-C_{24}\right)+$ $\left(2 C_{13}-H_{1}-H_{3}\right)+\left(2 C_{24}-H_{2}-H_{4}\right)+\left(2 C_{23}-H_{2}-H_{3}\right)$, and that's the sum of the $4 \mathrm{Tu}$ relation and 3 neck cutting relations.

Thus either one of $3 S_{1}$ or $3 S_{2}$ could have served as an alternative foundation for our theory replacing $4 \mathrm{Tu}$.

\subsection{Khovanov's $c$}

In his original paper [10] on categorification Khovanov introduced a more general knot homology theory, defined over the polynomial ring $\mathbb{Z}[c]$ where $\operatorname{deg} c=$ 2. The more general theory is defined using a functor, which we will call $\mathcal{F}_{c}$, which is similar to the functor $\mathcal{F}$ of Definition 7.1

$$
\begin{aligned}
& \epsilon_{c}:\left\{1 \mapsto v_{+}\right. \\
& \eta_{c}:\left\{\begin{array}{l}
v_{+} \mapsto-c \\
v_{-} \mapsto 1
\end{array}\right. \\
& \Delta_{c}:\left\{\begin{array}{l}
v_{+} \mapsto v_{+} \otimes v_{-}+v_{-} \otimes v_{+}+c v_{-} \otimes v_{-} \\
v_{-} \mapsto v_{-} \otimes v_{-}
\end{array}\right. \\
& m_{c}: \begin{cases}v_{+} \otimes v_{-} \mapsto v_{-} & v_{+} \otimes v_{+} \mapsto v_{+} \\
v_{-} \otimes v_{+} \mapsto v_{-} & v_{-} \otimes v_{-} \mapsto 0 .\end{cases}
\end{aligned}
$$

This more general theory remains little studied, and unfortunately, it doesn't fit inside our framework. The problem is that $\mathcal{F}_{c}$ does not satisfy the $S$ and the $4 \mathrm{Tu}$ relations, and hence it does not descend from $\mathcal{C}_{o b}{ }^{3}$ to $\mathcal{C}_{o b}{ }_{/ l}^{3}$.

It is natural to seek for replacements for $S$ and $4 T u$ which are obeyed by $\mathcal{F}_{c}$ and to try to repeat everything using those replacements. A natural replacement for the $S$ relation may be the relation $S_{c}: \Theta=-c$, and the following relation may be a suitable replacement for $4 T u$ (in its $3 S_{2}$ guise; see Section [11.4):

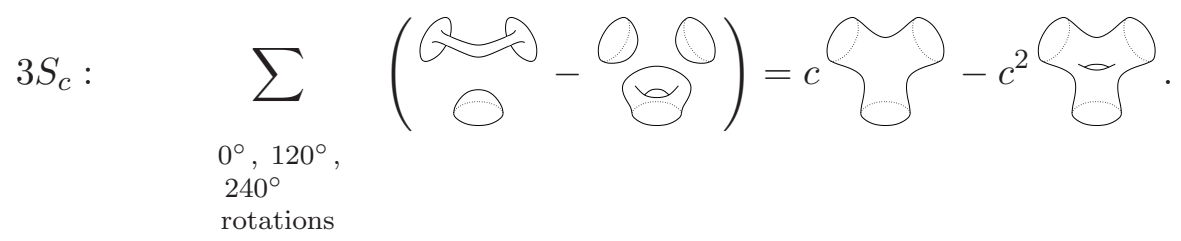

Indeed, one may verify that $\mathcal{F}_{c}$ satisfies $S_{c}$ and $3 S_{c}$. 
Problem 11.3 Is there a parallel for our theory with $S_{c}$ and $3 S_{c}$ replacing $S$ and $4 \mathrm{Tu}$ ?

We've been able to prove invariance under $R 1$ using $S_{c}$ and $3 S_{c}$, but not invariance under $R 2$ (one may hope that invariance under $R 2$ will imply invariance under $R 3$ as in Section 4.3. so only invariance under $R 2$ is really missing). Note, though, that the proof of Lemma 8.6. and hence of invariance under movie moves (Theorems 4 and 5 ), depend in a fundamental way on the $S$ relation. Hence there is no reason to expect that the theory defined by $\mathcal{F}_{c}$ would have an invariant extension to $\mathcal{C}_{0} b_{/ i}^{4}$. Indeed Jacobsson [7] has shown that such extension does not exist.

\subsection{Links on surfaces}

Almost everything done in this paper is local in nature, and so generalizes with no difficulty to links or tangles drawn on surfaces (more precisely, embedded in thickened surfaces; see Figure 17), in the spirit of [1]. The main challenge seems to be to figure out the full collection of "TQFTs" that can be applied in this case in order to get computable homology theories as in Section $\mathbf{7}$

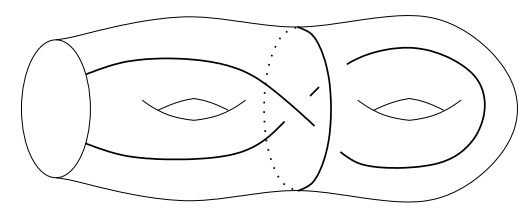

Figure 17: A 3-crossing 2-component (one closed, one open) 2-ended "tangle" drawn on a genus 2 surface with one boundary component $\Sigma_{1}^{1}$ (or embedded in $\Sigma_{1}^{1} \times I$ ).

\section{Glossary of notation}

We give a quick glossary of notation by section number:

[.] the formal Khovanov bracket, $Q$ the $\operatorname{cup} Q: \bigcirc \rightarrow \emptyset, 4.3$ 2.1.

[.] height shift in complexes, 4.3

$3 S_{i}$ the three-site relations, 11.4.

$\{\cdot\} \quad$ degree shift, 6 ,

, $\propto$ the cap $: \propto: \emptyset \rightarrow \bigcirc, 4.3$ $4 \mathrm{Tu}$ the four tube relation, 4.1.2 $\alpha$ an automorphism of a complex, 8.3 . 
$\Gamma$ the cone of a morphism, 4.3.

$\partial T$ boundary of $T$, . 3

$\Delta$ a part of $\mathcal{F}, \mathbf{Z}$

$\epsilon$ a part of $\mathcal{F}, \mathbf{Z}$

$\eta$ a part of $\mathcal{F},[$

$\xi_{i} \quad$ an edge of a cube, 2. 6 .

$\Xi$ the trace group, 10.2 .

$\Xi_{k}$ the trace group of $\mathcal{C}_{0} b_{0 / l}^{3}(k)$, 10.3 .

$\sigma$ the isomorphism $\mathcal{S}_{k} \rightarrow \Xi_{k}$, 10.3

$\tau$ a trace or a Lefschetz number 10.1 .

$\tau_{\star}$ the universal trace, 10.2

$\Phi$ a morphism of planar algebras, 1.1 .2

$\chi \quad$ Euler characteristic, 6.

$\chi_{\tau} \quad \tau$-Euler characteristic, 10.1

$\chi_{\star}$ the universal Euler characteristic, 10.2

$\Psi \quad$ a morphism of complexes, 4.3.

$\Omega^{r}$ a chain group, 3 ,

$A$ an Abelian group, 10.1

$\mathcal{A}$ an Abelian category, $\mathbf{7}$

$\mathcal{A l g}$ the 'algebraic picture', 1.1.2

$B$ a finite set of points on $S^{1}, 3$

$c$ Khovanov's $c$, 11.5.

$C$ a cobordism, 1

$\mathcal{C}$ a category, 3 ,

$\mathcal{C}_{o b} b^{3}$ either $\mathcal{C}_{o b} b^{3}(\emptyset)$ or $\mathcal{C}_{o b}(B)$, 3.

$\operatorname{Cob}^{3}(\emptyset)$ 3D cobordisms, no vertical boundary, 3 ,
$\mathcal{C}_{o b}{ }^{3}(B) \quad 3 \mathrm{D}$ cobordisms, vertical boundary $B \times I, 3$

$\mathcal{C}_{o b}{ }^{3}$ the quotient of $\mathcal{C}_{o b} b^{3}$ by $S, T$ and $4 T u$. Likewise for $\mathcal{C}_{0} b_{l l}^{3}(\emptyset)$ and $\mathcal{C}_{o b}{ }^{3}(B)$. 4.1.2.

$\mathcal{C}_{o b} b_{0 / l}^{3}$ a variant of $\mathcal{C}_{o b}{ }^{3}, 10.3$,

$\mathcal{C} o b_{\bullet}^{3}$ dotted cobordisms, 11.2

$\mathrm{Cob}_{\bullet / l}^{3}$ dotted cobordisms modulo local relations, 11.2.

$\mathcal{C}_{o b}{ }^{4}$ either $\mathcal{C}_{o b} b^{4}(\emptyset)$ or $\mathcal{C}_{o b} b^{4}(B)$, 8.

$\operatorname{Cob}^{4}(\emptyset) \quad 4 \mathrm{D}$ cobordisms, no vertical boundary, 8

$\operatorname{Cob}^{4}(B) \quad 4 \mathrm{D}$ cobordisms, vertical boundary $B \times I, 8$

$\mathcal{C o b}_{/ i}^{4}$ the quotient of $\mathcal{C o b}^{4}$ by isotopies. Likewise for $\mathcal{C}_{o b}{ }_{i i}^{4}(\emptyset)$ and $\operatorname{Cob}_{/ i}^{4}(B)$. 8

$d, d^{r}$ differentials, 3 .

$D$ a planar arc diagram, 5 ]

$\operatorname{dim}_{\tau}$ the $\tau$-dimension, 10.1.

$\operatorname{dim}_{\star}$ the universal dimension, 10.2 .

$F, G$ morphisms (mainly between complexes), 3 .

$\mathcal{F}$ a TQFT functor, 1.1.2 7

$\mathbb{F}_{2}$ the two element field, 9.

$g$ genus, 9.1

$\mathcal{G}_{\geq j} \quad$ a filtration, 9.3 .

$h, h^{r}$ homotopies, 4.1.1.

$H$ a handle, 9.3

$j$ a specific degree, 9.3

$\hat{J}, J$ the Jones polynomial, 7 . 10.

K a knot, 2, 1 . 
Kh Khovanov homology, 1 , 6 .

$K h_{0}$ Khovanov homology on movies, 8.2 .

$\operatorname{Kob}(\cdot) \operatorname{Kom}\left(\operatorname{Mat}\left(\mathcal{C}_{o b}^{3}{ }_{l l}\right)\right)$, complexes made of cobordisms, 4.2 .

$\mathrm{Kob}_{/ h}(\cdot) \operatorname{Kob}(\cdot)$ modulo homotopy, 4.2

$\mathrm{Kob} / \pm$ projectivized Kob, 8 .

$\mathrm{Kob}_{/ \pm h}$ projectivized $\mathrm{Kob} / h, 8$

$\operatorname{Kom}(\cdot)$ complexes over a category, 3 ,

$\operatorname{Kom}_{/ h}(\cdot) \operatorname{Kom}(\cdot)$ modulo homotopy, 3 .

$m$ a part of $\mathcal{F}, \mathbf{7}$

Mat(.) matrices over a category, 3

$\mathrm{MM}_{i}$ movie moves, 8.3

$n$ number of crossings, 2 . 1.

$n_{ \pm}$number of \pm crossings, 2 2 .

$L, L_{i} \quad$ links, 1

$\mathcal{O}_{i}$ objects in a category, 6.

$\mathcal{P}(k)$ the sets making up an unoriented planar algebra, 5 .

$\mathcal{P}(s)$ the sets making up an oriented planar algebra, 5 . $q$ a formal variable, 10.2

$r$ homological degree, 3 .

Ri Reidemeister moves, 4.2

$S$ a smoothing, 10.3

$S$ the sphere relation, 4.1.2

$\mathcal{S}_{k}$ a skein module, 10.3.

$T$ a tangle or a tangle diagram, 1.1.1.

$T$ the torus relation, 4.1.2

$\mathcal{T}^{0}(k)$ unoriented $k$-ended tangle diagrams, 5 .

$\mathcal{T}^{0}(s)$ oriented $k$-ended tangle diagrams, 5 .

$\mathcal{T}(k)$ unoriented $k$-ended tangles, 5 .

$\mathcal{T}(s)$ oriented $k$-ended tangles, 5

Top the 'topological picture', 1.1 .2 .

$v_{ \pm}$the generators of $V, \mathbf{7}$

$V$ the $\mathbb{Z}$-module for a circle, $\mathbf{7}$

$X$ a crossing, 5 , 8.3

$\mathbb{Z}_{(2)} \quad \mathbb{Z}$ localized at $2,9.1$

$\mathbb{Z}$ Mod $\mathbb{Z}$-modules, $\mathbf{7}$

\section{References}

[1] M M Asaeda, J H Przytycki, A S Sikora, Khovanov homology of links in I-bundles over surfaces, arXiv:math.QA/0403527

[2] MM Asaeda, JH Przytycki, A S Sikora, Categorification of the Kauffman bracket skein module of I-bundles over surfaces, Algebr. Geom. Topol. 4 (2004) 1177-1210 MathReview

[3] D Bar-Natan, On Khovanov's categorification of the Jones polynomial, Algebr. Geom. Topol. 2 (2002) 337-370 MathReview 
[4] J S Carter, M Saito, Knotted surfaces and their diagrams, Mathematical Surveys and Monographs 55, American Mathematical Society, Providence, RI (1998) MathReview

[5] J S Carter, M Saito, S Satoh, Ribbon-moves for 2-knots with 1-handles attached and Khovanov-Jacobsson numbers, arXiv:math.GT/0407493

[6] M Jacobsson, An invariant of link cobordisms from Khovanov homology, Algebr. Geom. Topol. 4 (2004) 1211-1251 MathReview

[7] M Jacobsson, Khovanov's conjecture over $\mathbb{Z}[c]$, arXiv:math.GT/0308151

[8] V Jones, Planar algebras, I, New Zealand Journal of Mathematics (to appear) arXiv:math.QA/9909027

[9] L H Kauffman, State models and the Jones polynomial, Topology 26 (1987) 395-407 MathReview

[10] M Khovanov, A categorification of the Jones polynomial, Duke Math. J. 101 (2000) 359-426 MathReview

[11] M Khovanov, A functor-valued invariant of tangles, Algebr. Geom. Topol. 2 (2002) 665-741 MathReview

[12] M Khovanov, Patterns in knot cohomology. I, Experiment. Math. 12 (2003) 365-374 MathReview

[13] M Khovanov, An invariant of tangle cobordisms, Trans. Amer. Math. Soc. (to appear) arXiv:math.QA/0207264

[14] E S Lee, On Khovanov invariant for alternating links, arXiv:math.GT/0210213

[15] J H Przytycki, Fundamentals of Kauffman bracket skein modules, Kobe J. Math. 16 (1999) 45-66 MathReview

[16] J A Rasmussen, Khovanov homology and the slice genus, e-print (2004) arXiv:math.GT/0402131

[17] J A Rasmussen, Khovanov's invariant for closed surfaces, e-print (2005) arXiv:math.GT/0502527

[18] R Scharein, KnotPlot, http://www.pims.math.ca/knotplot/

[19] A N Shumakovitch, Torsion of the Khovanov homology, e-print (2004) arXiv:math.GT/0405474

[20] J Stallings, Centerless groups - an algebraic formulation of Gottlieb's theorem, Topology 4 (1965) 129-134 MathReview

[21] O Viro, Remarks on definition of Khovanov homology, e-print (2002) arXiv:math.GT/0202199

[22] S Wolfram, The Mathematica ${ }^{\circledR}$ book, Wolfram Media, Inc. Champaign, IL (1999) MathReview 